\title{
Aprendizagem Empreendedora Conhecendo o Passado e Vislumbrando o Futuro
}

http://dx.doi.org/10.21527/2237-6453.2019.49.247-275

Recebido em: 22/11/2017

Aceito em: $23 / 5 / 2019$

Sérgio Vogt, ${ }^{1}$ Yara Lucia Mazziotti Bulgacov ${ }^{2}$

\section{RESUMO}

Os questionamentos que surgiram no campo de estudo do empreendedorismo no final da década de 80 incentivaram a realização de pesquisas que buscavam o entendimento do aprendizado no processo de empreender. Como resultado, inaugurou-se uma nova temática denominada de Aprendizagem Empreendedora (AE), que trouxe contribuições para a compreensão do empreendedorismo como processo. Para descobrir como estão sendo conduzidos os estudos sobre esse tema no cenário nacional e internacional, realizou-se neste artigo um levantamento sistemático da produção de conhecimento em diferentes bases acadêmicas de dados. O trabalho foi realizado sob uma perspectiva histórica, permitindo que os resultados apontassem para as possibilidades de pesquisas ainda não exploradas, principalmente no que se refere ao desenvolvimento de aproximações empíricas sobre a aprendizagem empreendedora na esfera nacional. Assim, visto que esse artigo não olha somente para o passado, mas aponta para o futuro, salienta-se que o entendimento da aprendizagem empreendedora é um processo inacabado no que respeita às pesquisas e sugere-se que se busque avançar na compreensão do processo de aprendizagem dos empreendedores investigando-o sob a perspectiva das práticas sociais.

Palavras-chave: Aprendizagem empreendedora. Empreendedorismo. Aprendizagem. Levantamento sistemático da literatura.

\section{ENTREPRENEURIAL LEARNING: KNOWING THE PAST AND SIGHTING THE FUTURE}

\section{ABSTRACT}

The questions that arose in the field of entrepreneurship in the late eighties encouraged researches to seek an understanding of learning in the process of undertaking. As a result, a new theme called Entrepreneurial Learning (EL) emerged, bringing contributions to the understanding of entrepreneurship as a process. To find out how the studies on this topic are being carried out in the national and international scene, a systematic literature review, within a historical perspective, was carried out in different academic databases. The results point out the possibilities of research not yet explored, mainly in the development of empirical studies about entrepreneurial learning in the national sphere. This research does not look only to the past, but points to the future, emphasizing that the understanding of entrepreneurial learning is an unfinished process. Therefore, it is proposed to advance the research of the entrepreneurs' learning process through the perspective of social practices.

Keywords: Entrepreneurial learning. Entrepreneurship. Learning. Systematic literature review.

\footnotetext{
${ }^{1}$ Mestre em Administração pela Universidade Federal do Paraná (UFPR). Doutorando em Administração pela Universidade Positivo (UP). sergiovogt@gmail.com

${ }^{2}$ Doutora em Educação pela Universidade Estadual de São Paulo (Unesp). Professora da Universidade Positivo (UP). ybulgacov@gmail.com
} 
Enquanto campo de estudo o empreendedorismo não é recente e já foi explorado utilizando-se de diferentes perspectivas (CAMPOS; PARELLADA; PALMA, 2012). Ele surgiu dentro de uma vertente econômica (SAY, 1971; SCHUMPETER, 1997) e se expandiu com o auxílio da corrente humanista, principalmente da Psicologia (MCCLELLAND, 1972). Sua importância e relevância na área acadêmica ainda são destacadas em virtude da sua significância não só na economia, mas no desenvolvimento social e cultural (RAE, 2000; SHANE; VENKATARAMAN, 2000; BYGRAVE, 2009; RAE; WANG, 2015).

A ênfase como tema de estudo ganhou fôlego não somente em virtude do seu impacto nessas diferentes esferas da realidade, mas também pelo surgimento de um novo horizonte de pesquisa apontado por William B. Gaertner no final da década de 80 . A perspectiva de estudo sugerida pelo autor proporcionou um novo olhar sobre o campo, possibilitando que o empreendedorismo fosse analisado como um processo dinâmico e não mais estático (GARTNER, 1988; RAE; CARSWELL, 2000; SHANE; VENKATARAMAN, 2000; RAE; CARSWELL, 2001). Dessa maneira, os pesquisadores passaram a questionar como ocorre o processo do "tornar-se" empreendedor, diferente do que até então era observado, uma vez que o foco estava na questão do "ser" empreendedor.

Como resultado dessa mudança passou-se a defender a ideia de que a pesquisa acadêmica já havia trazido consideráveis contribuições sobre a perspectiva extrínseca do conhecimento em empreendedorismo, e que era momento de se aprofundar na compreensão do processo humano intrínseco. Assim, a partir da década de 90 e de forma mais intensa no início dos anos 2000, surgiram as primeiras pesquisas que focavam na busca do entendimento do processo de aprendizado em que os empreendedores se envolvem ao empreender (FESTERVAND; FORREST, 1993; MURPHY, 1993; SMILOR, 1997; YOUNG; SEXTON, 1997; DEAKINS; FREEL, 1998; RAE; CARSWELL, 1999; COPE; WATTS, 2000; RAE, 2000; RAE; CARSWELL, 2000; HANNON, 2001; MINNITI; BYGRAVE, 2001; RAE; CARSWELL, 2001).

A busca de compreensão sobre a aprendizagem do empreendedor inaugurou uma nova temática denominada de Aprendizagem Empreendedora (AE), que surgiu da relação entre as teorias sobre empreendedorismo e aprendizagem, principalmente a Aprendizagem Organizacional $(\mathrm{AO})$ quando considerada dentro da perspectiva de que são os indivíduos que aprendem no contexto organizacional (MINNITI; BYGRAVE, 2001; HARRISON; LEITCH, 2008; ERDÉLYI, 2010; WANG; CHUGH, 2014, 2015).

$A$ atenção dada à $A E$ como tema de estudo trouxe contribuições para o entendimento do empreendedorismo como processo e do desenvolvimento do empreendedor à medida que esse é o agente que empreende (YOUNG; SEXTON, 1997; RAE; CARSWELL, 2000; POLITIS, 2005; RAE, 2005a; HARRISON; LEITCH, 2008; RAE; WANG, 2015). O aparente crescimento nas pesquisas sobre a relação do "aprender" e "empreender" (e vice-versa) revelado pelo levantamento sistemático da literatura realizado por Wang e Chugh (2014), desencadeou o questionamento e o interesse em descobrir sobre como estariam sendo conduzidos os estudos sobre a $A E$, tanto no cenário internacional (LI) como nacional (LN). ${ }^{3}$

3 Esse escopo de pesquisa foi definido tendo em vista que o estudo realizado por Wang e Chugh (2014) abrange apenas a literatura international até o período de 2011. E na literatura nacional não existe um levantamento sistemático sobre a produção de conhecimento e sobre a aprendizagem empreendedora. 
Por consequência, foi realizado um levantamento sistemático da literatura sobre $\mathrm{AE}$ contemplando essas duas esferas (nacional e internacional), constituindo o objetivo deste trabalho revelar as possibilidades de pesquisas na literatura brasileira sobre a aprendizagem empreendedora ainda não exploradas. O mapeamento da literatura e a descrição dos resultados pretende mostrar uma visão geral do campo, enfatizando as diferentes fases pela qual o tema passou no decorrer dos anos e como tem sido explorado academicamente na atualidade.

Para tanto, as seções seguintes irão apresentar os aspectos teórico-históricos e metodológicos que conduziram o levantamento efetuado, divulgando os critérios utilizados para a realização da pesquisa. Posteriormente serão demonstrados os resultados obtidos, bem como as implicações para futuras pesquisas.

\section{APRENDIZAGEM EMPREENDEDORA: Surgimento}

Em meados dos anos 70 o campo do empreendedorismo como tema de pesquisa já havia se consolidado, principalmente dentro de uma vertente econômica (BAUMOL, 1968; SAY, 1971; KIRZNER, 1973), porém, nesse mesmo período, surgiu outra possibilidade de reflexão sobre a temática com a perspectiva humanista de Mcclelland (1972). Essa mudança, de uma perspectiva puramente econômica para um olhar mais humano sobre o empreendedorismo, pode ter motivado Lawrence Lamont (1972) a questionar o que os empreendedores aprendem por meio da experiência.

Até então a relação entre o aprender e o empreender não havia sido pesquisada na esfera acadêmica, como revela o levantamento sistemático da literatura realizado por Wang e Chugh (2014). O aprender (aprendizagem) e o empreender (empreendedorismo) eram áreas tratadas de forma separada e, conforme destacou Mark Easterby-Smith, essas duas temáticas "têm muito a ganhar se conversarem" (HARRISON; LEITCH, 2008 , p. xxii). Esse primeiro passo dado por Lamont, contudo, parece não ter despertado de forma imediata o interesse do campo, pois somente uma década depois Tucker Jr. (1981) unirá os temas da aprendizagem e do empreendedorismo ao abordar o ensino deste último. Após isso, novamente o campo de estudo se manteve adormecido nos anos seguintes.

Um novo despertar do interesse parece ter surgido quando Gartner (1988) levantou a questão de que as pesquisas sobre o(a) empreendedor(a) deveriam focar no que ele(a) faz e não em quem ele(a) é. Essa mudança de enfoque buscou incentivar a dinamicidade de um olhar processual sobre o fenômeno do empreender e também do tornar-se empreendedor. Como resultado, Scherer, Adams e Wiebe (1988) sugeriram que a Teoria da Aprendizagem Social fosse usada como uma base teórica para a pesquisa em empreendedorismo.

Um pouco depois, já no início da década de 90, apareceram alguns passos firmes em direção à apresentação da $\mathrm{AE}$ como um tema de pesquisa dentro da área acadêmica, primeiramente com Lant e Mezias (1990) ao colocarem novamente a temática da Aprendizagem Organizacional (AO) e do empreendedorismo, de forma conjunta, para responder às mudanças ambientais que algumas organizações sofrem. Foi principalmente com Festervand e Forrest (1993), porém, que apresentaram o conceito de Entre- 
preneurial Preparedness na busca de desenvolver um modelo que ofereça respostas à preparação empreendedora, que se avançou em direção ao crescimento e ao desenvolvimento da $A E$ como campo de pesquisa.

Embora nesse mesmo período o trabalho de Murphy (1993) já tenha utilizado a expressão "aprendizagem empreendedora", ao considerar que o ambiente organizacional imerso em mudanças demanda que o empreendedor tenha de buscar formas diferentes de agir e que isso envolve o aprendizado, foi somente no final dos anos 90 que a expressão passa a ser utilizada de forma explícita e recorrente como temática que identifica uma área de estudo.

Isso ocorreu primeiramente com Young e Sexton (1997), que investigaram o processo que permite que empreendedores (já atuantes) aprendam na medida em que gerenciam seus negócios. Sugere-se, assim, que os empreendedores aprendem de forma processual, dado que foi desenvolvido o que pode ser considerado uma das primeiras tentativas de descrição do processo de aprendizagem empreendedora. Em seguida, Deakins e Freel (1998) empregaram o termo na tentativa de verificar a contribuição da teoria da AO em pequenas e médias empresas e, posteriormente, com o trabalho de Rae e Carswell (1999), o interesse foi a busca do desenvolvimento de um modelo conceitual em AE.

No cenário nacional, Valdir Gomes (2000) destaca-se como o primeiro trabalho a abordar os aspectos da aprendizagem e do empreendedorismo. Ao elaborar um ensaio teórico para um evento nacional (Encontro de Estudos em Empreendedorismo e Gestão de Pequenas Empresas - Egepe), mesmo não utilizando a literatura internacional disponível sobre $A E$, o autor emprega a literatura disponível em aprendizagem e empreendedorismo para dar ênfase ao ensino do empreendedorismo.

Posterior a esse trabalho, Guardani e Romito (2008) apresentaram um artigo no mesmo Congresso, porém em uma edição oito anos mais tarde, que se destaca como o primeiro trabalho empírico da temática na esfera nacional. Nele, os autores buscaram analisar como os empreendedores aprendem a administrar seus negócios. Ressalta-se que, também nessa pesquisa, não foram utilizadas as bases teóricas existentes sobre $A E$. Isso mudou somente a partir de 2010, quando o trabalho empírico de Zampier empregou uma base teórica fundamentada na literatura internacional sobre AE (ZAMPIER, 2010).

Uma vez realizado esse sucinto olhar histórico do surgimento da $A E$ como tema de estudo, percebe-se que houve um crescimento do interesse e da pesquisa até o final da década de 90 na literatura internacional, diferente do cenário nacional. Assim, para que se evidencie essa primeira fase da AE e se argumente sobre o desenvolvimento do tema nos anos seguintes, serão apresentados na seção a seguir os aspectos metodológicos que nortearam a realização do levantamento sistemático da literatura que dá base para esses argumentos.

\section{PROCEDIMENTOS METODOLÓGICOS}

Com o objetivo de investigar a produção de conhecimento sobre AE na esfera nacional (LN) e internacional (LI), foi realizado um levantamento sistemático na literatura, conforme Tranfield, Denyer e Smart (2003) e Denyer e Tranfield (2008), em diferentes bases acadêmicas de dados que possuíssem um número elevado de materiais indexados (como artigos de revistas e de eventos, livros, capítulos de livros e outros trabalhos acadêmicos), a fim de que se obtivesse um amplo resultado de referências sobre o tema. 
Desse modo, para a pesquisa com escopo internacional foram selecionadas as seguintes bases (listadas em ordem alfabética): EBSCO; Emerald Insight; JSTOR; Portal de Periódicos da Coordenação de Aperfeiçoamento de Pessoal de Nível Superior (Capes); ProQuest; SciELO; Science Direct; Scopus; Spell e Web of Science. A pesquisa para a revisão sistemática da Literatura Nacional (LN) empregou a expressão "Aprendizagem Empreendedora" e utilizou como critério de busca a expressão Entrepreneurial Learning nos títulos das publicações. Esse filtro foi estipulado considerando o interesse em identificar apenas os trabalhos que utilizaram esse tema de forma central, o que parece ficar evidente quando apresentado no título da pesquisa.

Optou-se por selecionar apenas os trabalhos que foram publicados na língua inglesa para o levantamento da literatura internacional. Com relação ao período de abrangência da pesquisa, foram delimitados aqueles que tivessem data de publicação até o ano de 2016. Não houve um período inicial definido, considerando que o objetivo era identificar quando ocorreram as primeiras publicações sobre a temática.

Sabendo-se da existência de possíveis duplicidades de trabalhos nos dados que foram obtidos, uma vez que um material pode e acaba sendo indexado em mais de uma das bases de dados consultadas, com o auxílio do software Endnote ${ }^{T M} X 8$ foram identificados e excluídos os textos duplicados. A escolha de realizar a pesquisa em um número elevado de bases acadêmicas busca, mesmo sabendo da grande quantidade de duplicações que iriam surgir, ampliar as possibilidades de identificação de referências que revelassem de fato o campo de estudo do tema em questão.

Após a seleção das publicações realizou-se uma revisão visual e individualizada de todos os trabalhos com o intuito de detectar algum tipo de inconsistência que pudesse não ter sido identificada na primeira verificação realizada pelo software, principalmente no que se refere à redação de nome de autor(es), de título da obra e também do local em que foram publicados. Assim, novamente foi efetuada a retirada de trabalhos que ainda constavam em duplicidade.

Durante essa fase também foi realizada a leitura dos resumos dos trabalhos para identificar se porventura alguma das referências não havia sido anexada ao resultado da pesquisa por possuir ligação indireta com a temática, pois mesmo tendo sido definido o critério na pesquisa da expressão Entrepreneurial Learning no título, apareceram resultados que não eram coerentes com o escopo estipulado. Assim, por conseguinte, também foram excluídos esses materiais.

A pesquisa para a revisão sistemática da Literatura Nacional (LN) empregou a expressão "Aprendizagem Empreendedora" e foi realizada observando-se os mesmos critérios utilizados na revisão internacional, porém além das mesmas bases de dados já citadas anteriormente, foram adicionadas outras fontes de pesquisa que visam a expandir a busca sobre o tema em bases que poderiam não estar indexadas nas utilizadas para a pesquisa internacional.

Dessa forma, foram adicionados o portal de eventos da Associação Nacional de Pós-Graduação e Pesquisa em Administração - Anpad - que compila os trabalhos que foram publicados nos eventos da Associação; a Revista de Empreendedorismo e Gestão de Pequenas Empresas - Regepe - que possui foco em empreendedorismo; o Encontro de Estudos sobre Empreendedorismo e Gestão de Pequenas Empresas - Egepe - que é o evento da Associação Nacional de Estudos em Empreendedorismo e Gestão de Pequenas Empresas - Anegepe, além do Google Acadêmico, que possui uma amplitude de busca em diferentes bases de dados, especialmente em periódicos não indexados nas bases de dados utilizadas. 
Assim, uma vez descritos os procedimentos empregados na seleção dos materiais para esta pesquisa, os resultados obtidos serão apresentados na próxima seção, a qual revela como se constituíram as diferentes fases em que a $\mathrm{AE}$ tem sido estudada nos cenários nacional e internacional.

\section{APRESENTAÇÃO DOS RESULTADOS E ANÁLISE}

Como primeiro resultado da pesquisa foram identificados 134 referências na literatura internacional e 21 na literatura nacional. Uma vez anexados ao Endnote ${ }^{T M}$, esses materiais compõem a base para a realização da análise a fim de que se obtenha um mapeamento detalhado do tema. A descrição de onde e quando as pesquisas em AE surgiram aparece quando realizada a distribuição desses resultados de acordo com o tipo de referência no decorrer dos anos. Para isso, as Tabelas 1 e 2 a seguir trazem essa representação.

Tabela 1 - Ano e tipo de material na Literatura Internacional

\begin{tabular}{|c|c|c|c|c|c|c|c|c|c|}
\hline Ano & Artigo & Evento & Tese & Ano & Artigo & Evento & Capítulo & Tese & Livro \\
\hline 1981 & 1 & & & 2008 & 3 & 1 & & & 1 \\
\hline 1998 & 1 & & & 2009 & 4 & 3 & & & \\
\hline 2000 & 4 & & & 2010 & 4 & 5 & 1 & & \\
\hline 2001 & 3 & & & 2011 & 6 & 4 & 1 & & \\
\hline 2002 & 1 & & & 2012 & 7 & 3 & 2 & & \\
\hline 2003 & 2 & & & 2013 & 6 & 2 & 1 & & \\
\hline 2004 & 4 & 2 & & 2014 & 6 & 4 & 4 & 1 & 1 \\
\hline 2005 & 6 & & & 2015 & 6 & 7 & & 1 & 1 \\
\hline 2006 & 1 & & 1 & 2016 & 6 & 1 & 1 & & \\
\hline 2007 & 2 & 2 & 1 & TOTAL & 73 & 34 & 10 & 4 & $3^{4}$ \\
\hline
\end{tabular}

Tabela 2 - Ano e tipo de material na Literatura Nacional

\begin{tabular}{c|c|c|c|c} 
Ano & Artigo & Evento & Dissertação & Tese \\
\hline 2000 & & 1 & & \\
\hline 2008 & & 1 & & \\
\hline 2010 & & & 1 & \\
\hline 2011 & 1 & & & \\
\hline 2012 & 1 & 1 & 1 & \\
\hline 2013 & & 1 & 1 & \\
\hline 2014 & 1 & 2 & 1 & \\
\hline 2015 & 3 & 1 & & 1 \\
\hline 2016 & 2 & 1 & & \\
\hline TOTAL & 8 & 8 & 4 & 1
\end{tabular}

\footnotetext{
${ }^{4}$ Do total de 134 trabalhos identificados, além dos 10 capítulos que foram publicados em livros diversos e que são descritos na tabela 1, outros 10 fazem parte de dois livros já apontados aqui. Por isso o total de trabalhos após realizada a divisão de acordo com o ano e tipo é de 124 trabalhos. Ou seja, não foram considerados esses últimos dez capítulos de livros, uma vez que foram considerados os livros nos quais eles foram publicados.
} 
Após a apresentação desses dados, em que é possível identificar o mapeamento da produção de conhecimento sobre aprendizagem empreendedora no decorrer do período estipulado e perceber a distribuição dos diferentes materiais produzidos, a seção a seguir propõe-se a discutir a consolidação e o crescimento desse campo de estudo pelo olhar mais detalhado sobre essas referências.

\section{A Consolidação e o Crescimento do Campo de Estudo: diferentes fases da temática}

Em uma observação minuciosa sobre essa gama de materiais disponíveis em Aprendizagem Empreendedora é possível definir determinados períodos da produção acadêmica de conhecimento nessa área. Na busca de evidenciar essas diferentes fases utilizou-se para fins de classificação a unidade de tempo - ano.

Faz-se necessário, porém, esclarecer que se emprega esse critério apenas como forma de sistematizar a leitura realizada pelos autores da pesquisa sobre esse campo, uma vez que se reconhece que não se pode afirmar com propriedade que um determinado campo de estudo passe por mudanças em períodos de tempo bem delimitados e que seja possível estipular de forma tão específica esses períodos. Defende-se aqui, com essa categorização, que o que ocorre são momentos e eventos que geram no observador da literatura a impressão de uma mudança na medida em que o tempo passa e a produção de conhecimento cresce.

Dito isso, as seções seguintes dedicam-se a apresentar e descrever as diferentes fases que foram identificadas e definidas com base nos resultados dos trabalhos apresentados no levantamento sistemático da literatura.

\section{A Primeira Fase da AE}

A contar do surgimento, o crescimento e a consolidação da pesquisa da Aprendizagem Empreendedora continuaram a partir dos anos 2000. Surgiram nessa época trabalhos como o de Rae (2000), que além de estimular o debate entre os acadêmicos sobre o uso da metodologia da narrativa como uma construção de significado na investigação do desenvolvimento da compreensão do empreendedorismo, também propôs um modelo conceitual em AE. No mesmo ano, baseando-se em Young e Sexton (1997), esse autor, em parceria com Mary Carswell, mantendo a mesma ênfase do seu trabalho anterior, busca avançar no desenvolvimento desse modelo conceitual (RAE; CARSWELL, 2000). Nesse mesmo período também surgiu o trabalho de Cope e Watts (2000) explorando o learning by doing, em que a experiência é vista como fonte do aprendizado. Tanto Jason Cope, assim como David Rae, serão considerados expoentes sobre a temática nos próximos anos.

Na sequência surgiu o texto de Sullivan (2000), que utilizou os trabalhos anteriores de Rae e de Deakins e Freel (1998) em sua base teórica. A publicação desse artigo baseou-se em um texto apresentado em um congresso no ano anterior, que já havia sido utilizado como base teórica para autores como Jason Cope e Gerald Watts. Ou seja, naquele período iniciou-se a construção encadeada da pesquisa que visava a se aprofundar na temática e para isso utilizavam-se os fundamentos firmados anteriormente. 
Essa época também foi marcada pelo surgimento de trabalhos que, assim como foi com Gartner (1988), geraram um novo ânimo ao campo do empreendedorismo, reforçando a necessidade de se avançar sobre a aprendizagem do empreendedor. Um desses trabalhos foi o de Shane e Venkataraman (2000), que descreveu o tema do empreendedorismo como uma promessa de campo de estudo, especialmente ao enfatizar que empreender refere-se à exploração de oportunidades. Outro trabalho foi de Bygrave e Minniti (2000), que explorou a dinâmica social do empreendedorismo, incentivando, um pouco depois, a elaboração de um modelo dinâmico da Aprendizagem Empreendedora (MINNITI; BYGRAVE, 2001).

Nos anos que seguem surgiram outros materiais que se dedicaram ao debate da Aprendizagem Empreendedora, com destaque para o trabalho realizado em conjunto por autores que já haviam aparecido como sendo uns dos primeiros a abordarem o tema, como Deakins, Sullivan e Whittam (2002). Esse período também é marcado pelo aparecimento do segundo trabalho de Jason Cope (COPE, 2003), além de outros, como o de Erikson (2003), Qin (2004), Qin e Bao (2004), Taylor e Thorpe (2004) e Wee (2004), assim como pelo surgimento dos novos trabalhos de David Rae (2004b), especialmente Rae (2004a) em que o autor introduz um novo modelo para a compreensão da aprendizagem empreendedora.

Dessa forma, em virtude daquilo que estaria por vir nos próximos anos, conclui-se "a primeira fase" no que se refere à produção de conhecimento sobre a aprendizagem empreendedora.

\section{A Segunda Fase da AE}

Sendo esse o cenário até 2004, posterior a esse período, a partir do ano de 2005, verifica-se uma nova fase - a segunda da Aprendizagem Empreendedora. Esse ano pode ser considerado um marco para o avanço nos estudos sobre a $A E$, pois foi o momento em que a temática realizou um grande salto. Isso ocorreu não apenas em virtude do surgimento de novos artigos (Rae, 2005a, b) que almejavam a busca pelo progresso do modelo proposto anteriormente, ou então pelo debate sobre o tema sugerido por Cope e Hamilton (2005) e Cope e Pittaway (2005) no British Academy of Management Conference, mas essencialmente pelo fato de a revista Entrepreneurship Theory and Practice (ET\&P) publicar em julho uma edição especial sobre AE.

Nessa edição especial, editada por Richard T. Harrison e Claire M. Leitch (HARRISON; LEITCH, 2005), que culminou com a publicação do primeiro livro sobre a temática três anos mais tarde (HARRISON; LEITCH, 2008), o destaque pode ser atribuído aos artigos de Cope (2005), que sugere que se avance em direção à dinâmica da aprendizagem em empreendedorismo, e de Politis (2005), que apresentou um novo framework conceitual sobre a $A E$, lançando-se como uma proeminente acadêmica nesse campo de estudos com trabalhos próprios e parcerias com outros autores (POLITIS, 2008; GABRIELSSON; POLITIS, 2012; EL-AWAD; GABRIELSSON; POLITIS 2017; TOUTAIN et al., 2017).

Os anos subsequentes, ainda antes da publicação desse primeiro livro sobre o tema, foram marcados pelo desenvolvimento de alguns trabalhos de autores já conhecidos no campo pela sua dedicação à pesquisa em $A E$, como Rae (2006) e Pittaway e Cope (2007). As primeiras teses de Doutorado com ênfase no tema foram 
identificadas nessa mesma época (SANZ-VELASCO, 2006; DUFFY, 2007). Novos debates foram realizados em congressos (DENG; WANG, 2007; XIAO; ZHUANG; CAO 2007) e outros artigos foram publicados em periódicos por autores até então não conhecidos dentro desse campo de pesquisa (MAN, 2006; BERGLUND; HELLSTRÖM; SJÖLANDER 2007).

Nesse resgate histórico, o ano de 2008 se destaca, não apenas pela continuação dos debates acadêmicos ocorridos em eventos (COPE, 2008; COPE; CAVE; ECCLES, 2008; DENG; WANG, 2008), ou pela produção de novos artigos científicos publicados em revistas (HUOVINEN; TIHULA, 2008; LIANG; DUNN, 2008; PÁSTOR; TAYLOR; VERONESI, 2008), mas principalmente pela publicação do livro exclusivo ao tema intitulado: Entrepreneurial Learning: Conceptual frameworks and applications (HARRISON; LEITCH, 2008), que revisou a literatura disponível em $A E$ até aquele momento.

Essa obra foi desenvolvida com a finalidade de consolidar o conhecimento até então alcançado sobre $A E$. O resultado obtido revelou que não havia uma identificação clara (nem um aceite) pela comunidade acadêmica de um corpo teórico e de metodologias que contribuíssem para a construção de uma teoria da Aprendizagem Empreendedora. Assim, havia um desafio a ser atingido: avançar em direção à construção, não somente de uma teoria unificada sobre $A E$, mas da exploração do tema de forma profunda e abrangente (HARRISON; LEITCH, 2008).

Esses autores, após relatarem a dificuldade em se identificar e estabelecer uma perspectiva integradora em $A E$, sugeriram que futuras pesquisas deveriam reconhecer essa diversidade e "construir a partir disso" (HARRISON; LEITCH, 2008, p. 18). Dessa forma, com a publicação desse livro e a partir do seu conteúdo, inaugurou-se um novo marco no estudo da Aprendizagem Empreendedora. Isso ocorreu pelo fato de que, além da revisão apresentada sobre o campo de estudo, também foram introduzidas novas sugestões de pesquisa sobre o tema para serem seguidas em futuros trabalhos.

\section{A Terceira Fase da AE}

Em consequência dessas novas sugestões, pode-se afirmar que a literatura sobre o tema passaria a partir daquela época a uma nova etapa, a qual aqui é denominada de terceira fase da aprendizagem empreendedora. Os anos que se seguiram à publicação do livro foram marcados por um crescimento expressivo do número de publicações em $A E$ em comparação com os anos anteriores, principalmente de natureza empírica, mas ainda nada que revolucionaria o que já estava sendo feito. Acredita-se que a produção de conhecimento, ocorrida posteriormente à publicação do livro e da existência de bases teóricas consolidadas, buscou apenas empregar aquilo que até então havia sido produzido e estava disponível sobre a Aprendizagem Empreendedora a fim de "testar" empiricamente esse corpo teórico acessível.

O período que se estende em torno dos cinco próximos anos - de 2009 até meados de 2014 - é caracterizado por uma grande produção de material em AE. Nesse intervalo de tempo surgiu um número considerável de capítulos de livros dedicados ao tratamento do tema, conforme quadro a seguir, principalmente em livros denominados Handbooks. 
Quadro 1 - Referências de capítulos de livros da terceira fase da AE

\begin{tabular}{|c|c|c|}
\hline Autor(es) & Título & Ano \\
\hline $\begin{array}{l}\text { Rae, David } \\
\text { Gee, Simon } \\
\text { Moon, Robert }\end{array}$ & $\begin{array}{l}\text { The role of an entrepreneurial learning team in creating an enter- } \\
\text { prise culture in a university }\end{array}$ & 2010 \\
\hline $\begin{array}{l}\text { Dickel, Petra } \\
\text { Andree, Helga }\end{array}$ & $\begin{array}{l}\text { Entrepreneurial learning in energy technology start- ups: A case } \\
\text { study in the biogas market }\end{array}$ & 2011 \\
\hline $\begin{array}{l}\text { Gabrielsson, Jonas } \\
\text { Politis, Diamanto }\end{array}$ & $\begin{array}{c}\text { Entrepreneurial learning and innovation: Building entrepreneur- } \\
\text { ial knowledge from career experience for the creation of new } \\
\text { ventures }\end{array}$ & 2012 \\
\hline Zhang, Xiaoxia & $\begin{array}{l}\text { The impact of entrepreneurial learning on entrepreneurial per- } \\
\text { formance: the mediating role of entrepreneurial competencies }\end{array}$ & 2012 \\
\hline $\begin{array}{l}\text { Hadjielias, Elias } \\
\text { Hamilton, Eleanor } \\
\text { Howorth, Carole }\end{array}$ & $\begin{array}{l}\text { Entrepreneurial learning in the family management group: A so- } \\
\text { cial organizational learning perspective }\end{array}$ & 2013 \\
\hline Rae, David & $\begin{array}{l}\text { Towards a momentary perspective in entrepreneurial learning } \\
\text { and creativity }\end{array}$ & 2014 \\
\hline Forbes, Daniel P. & The infrastructure of entrepreneurial learning & 2014 \\
\hline $\begin{array}{l}\text { Kwan, Diana S. } \\
\text { Yu, Fu-Lai Tony }\end{array}$ & $\begin{array}{l}\text { Entrepreneurial learning and capabilities development of a man- } \\
\text { ufacturing firm in China: The case of the Haier Group }\end{array}$ & 2014 \\
\hline $\begin{array}{l}\text { Van Vuuren, Win } \\
\text { et al. }\end{array}$ & $\begin{array}{c}\text { Entrepreneurial learning and the IBM universities business chal- } \\
\text { lenge: an experiential learning perspective }\end{array}$ & 2014 \\
\hline
\end{tabular}

Fonte: Elaborado pelos autores (2017).

Nesse período também foram apresentados diversos artigos em congressos, conforme quadro a seguir, que sinalizam um movimento da academia em direção às discussões relacionadas à $A E$.

Quadro 2 - Referências de artigos de eventos da terceira fase da AE

\begin{tabular}{|l|c|c|c|}
\hline \multicolumn{1}{|c|}{ Autor(es) } & Título & Evento & Ano \\
\hline $\begin{array}{l}\text { Chen, Wen- } \\
\text { ting } \\
\text { Li, Xin-chun }\end{array}$ & $\begin{array}{c}\text { Entrepreneurial Learning in New } \\
\text { Ventures: Dimensions Developing } \\
\text { and Structure Testing }\end{array}$ & $\begin{array}{c}\text { International Conference on Man- } \\
\text { agement Science \& Engineering }\end{array}$ & 2009 \\
\hline $\begin{array}{l}\text { Lee, Kiefer } \\
\text { Marriott, Sue } \\
\text { Lowe, Robin }\end{array}$ & $\begin{array}{c}\text { Promoting and Evaluating Entre- } \\
\text { preneurial Learning: Assessing the } \\
\text { Effectiveness of an Enquiry-Based } \\
\text { Approach }\end{array}$ & $\begin{array}{c}\text { 4th European Conference on Entre- } \\
\text { preneurship and Innovation }\end{array}$ & 2009 \\
\hline $\begin{array}{l}\text { Wei, Jiang } \\
\text { Gong, Limin } \\
\text { Liu, Chuwen }\end{array}$ & $\begin{array}{c}\text { Entrepreneurial Learning Based } \\
\text { on Critical Incidents: Influences of } \\
\text { Learning Styles }\end{array}$ & $\begin{array}{c}\text { International Conference on Strate- } \\
\text { gic Management }\end{array}$ & 2009 \\
\hline $\begin{array}{l}\text { Schout, Henk } \\
\text { Harkema, } \\
\text { Saskia }\end{array}$ & $\begin{array}{c}\text { Entrepreneurial Learning: Practice } \\
\text { as a Source for Learning and Busi- } \\
\text { ness Success }\end{array}$ & $\begin{array}{c}\text { 5th European Conference on Inno- } \\
\text { vation and Entrepreneurship }\end{array}$ & 2010 \\
\hline $\begin{array}{l}\text { Sjoevoll, Jarle } \\
\text { Pedersen, Ove }\end{array}$ & $\begin{array}{c}\text { Links between Educational Pro- } \\
\text { grammes, Local Recourses and En- } \\
\text { trepreneurial Learning }\end{array}$ & $\begin{array}{c}\text { 3rd International Conference of } \\
\text { Education, Research and Innovation }\end{array}$ & 2010 \\
\hline $\begin{array}{l}\text { Wilkinson, } \\
\text { Michael }\end{array}$ & $\begin{array}{c}\text { A Sociocultural-Historical Perspec- } \\
\text { tive on Entrepreneurial Learning: } \\
\text { The Case of a Novice Entrepreneur }\end{array}$ & $\begin{array}{c}\text { 6th International Seminar Quality } \\
\text { Management in Higher Education }\end{array}$ & 2010 \\
\hline $\begin{array}{l}\text { Xu, Jiang } \\
\text { Lu, Yanqiu }\end{array}$ & $\begin{array}{c}\text { Impacts of entrepreneurial network } \\
\text { on entrepreneurial learning }\end{array}$ & $\begin{array}{c}\text { 3rd International Conference on } \\
\text { Information Management, Innova- } \\
\text { tion Management and Industrial } \\
\text { Engineering - ICIII }\end{array}$ & 2010 \\
\hline
\end{tabular}




\begin{tabular}{|c|c|c|c|}
\hline $\begin{array}{l}\text { Zhiyuan, Li } \\
\text { Shukuan, Zhao }\end{array}$ & $\begin{array}{l}\text { Networking ties and entrepreneurial } \\
\text { learning: An empirical investigation } \\
\text { of Chinese enterprisers }\end{array}$ & $\begin{array}{c}\text { 2nd International Conference on } \\
\text { Networking and Digital Society - } \\
\text { ICNDS }\end{array}$ & 2010 \\
\hline $\begin{array}{l}\text { Chou, C. M. } \\
\text { et al. }\end{array}$ & $\begin{array}{c}\text { The influencing factors of student } \\
\text { teachers' entrepreneurial learning } \\
\text { behavior }\end{array}$ & $\begin{array}{c}\text { 4th International Joint Conference } \\
\text { on Computational Sciences and } \\
\text { Optimization - CSO }\end{array}$ & 2011 \\
\hline $\begin{array}{l}\text { Lagrosen, Ste- } \\
\text { fan } \\
\text { Josefsson, } \\
\text { Pernilla }\end{array}$ & $\begin{array}{l}\text { Social Media Marketing as an Entre- } \\
\text { preneurial Learning Process }\end{array}$ & $\begin{array}{l}\text { 4th Annual Euromed Conference of } \\
\text { the Euromed Academy of Business }\end{array}$ & 2011 \\
\hline $\begin{array}{l}\text { Moustaghfir, } \\
\text { Karim }\end{array}$ & $\begin{array}{c}\text { Entrepreneurial Learning: An Or- } \\
\text { ganizational Capability for Effective } \\
\text { Higher Education Services }\end{array}$ & $\begin{array}{l}\text { 6th International Forum on Knowl- } \\
\text { edge Asset Dynamics - IFKAD }\end{array}$ & 2011 \\
\hline $\begin{array}{l}\text { Roberts, David } \\
\text { Caton }\end{array}$ & $\begin{array}{l}\text { Rumination to Reflection: The Quest } \\
\text { to Re-Visit Entrepreneurial Learning }\end{array}$ & $\begin{array}{l}\text { 10th European Conference on Re- } \\
\text { search Methodology for Business } \\
\text { and Management Studies }\end{array}$ & 2011 \\
\hline $\begin{array}{l}\text { Akritidis, Loan- } \\
\text { nis } \\
\text { Kakouris, Alex- } \\
\text { andros }\end{array}$ & $\begin{array}{l}\text { Modelling the Style in Entrepreneur- } \\
\text { ial Learning from Experience }\end{array}$ & $\begin{array}{l}\text { 7th European Conference on Inno- } \\
\text { vation and Entrepreneurship - ECIE }\end{array}$ & 2012 \\
\hline \begin{tabular}{l|} 
Breuer, \\
Henning \\
Mahdjour, \\
Sarah \\
\end{tabular} & $\begin{array}{c}\text { Lean Venturing: Entrepreneurial } \\
\text { Learning to Model and Grow New } \\
\text { Business }\end{array}$ & $\begin{array}{c}\text { 5th Innovation Symposium - Stim- } \\
\text { ulating Innovation: Challenges for } \\
\text { Management, Science \& Technol- } \\
\text { ogy }\end{array}$ & 2012 \\
\hline $\begin{array}{l}\text { Lagrosen, } \\
\text { Yvonne } \\
\text { Lagrosen, Ste- } \\
\text { fan }\end{array}$ & $\begin{array}{l}\text { Entrepreneurial Learning for Qual- } \\
\text { ity and Innovation - a Study in the } \\
\text { Wellness Industry }\end{array}$ & $\begin{array}{l}\text { 7th European Conference on Inno- } \\
\text { vation and Entrepreneurship - ECIE }\end{array}$ & 2012 \\
\hline $\begin{array}{l}\text { Peng, Xiu-qing } \\
\text { Cai, Li }\end{array}$ & $\begin{array}{l}\text { The Relationship between Entrepre- } \\
\text { neurial Learning and Entrepreneur- } \\
\text { ial Knowledge }\end{array}$ & $\begin{array}{c}\text { 20th International Conference on } \\
\text { Management Science and Engi- } \\
\text { neering }\end{array}$ & 2013 \\
\hline $\begin{array}{l}\text { Yang, Jun-Ping } \\
\text { Chen, Ting- } \\
\text { ting } \\
\text { Tang, Lu-Bin } \\
\end{array}$ & $\begin{array}{c}\text { The effect of entrepreneurial net- } \\
\text { work on new venture growth: The } \\
\text { regulatory role of entrepreneurial } \\
\text { learning }\end{array}$ & $\begin{array}{c}\text { International Asia Conference on } \\
\text { Industrial Engineering and Manage- } \\
\text { ment Innovation - IEMI }\end{array}$ & 2013 \\
\hline $\begin{array}{l}\text { Fulford, Hea- } \\
\text { ther } \\
\text { Bailey, Moira }\end{array}$ & $\begin{array}{l}\text { Journals and Jottings on Entrepre- } \\
\text { neurial Learning Journeys }\end{array}$ & $\begin{array}{l}\text { European Conference on Innova- } \\
\text { tion and Entrepreneurship }\end{array}$ & 2014 \\
\hline Garlick, Ryan & $\begin{array}{l}\text { Entrepreneurial learning in a secure } \\
\text { E-Commerce course through creat- } \\
\text { ing competitive real-world sites }\end{array}$ & $\begin{array}{c}\text { International Conference on } \\
\text { E-Commerce, E-Business and E-Ser- } \\
\text { vice - EEE }\end{array}$ & 2014 \\
\hline $\begin{array}{l}\text { Marques, Ana } \\
\text { Paula } \\
\text { Moreira, Rita } \\
\text { Ramos, Sandra }\end{array}$ & $\begin{array}{c}\text { Higher Education, Stakeholders and } \\
\text { Collaborative Work for Entrepre- } \\
\text { neurial Learning }\end{array}$ & $\begin{array}{l}\text { 9th European Conference on Inno- } \\
\text { vation and Entrepreneurship - ECIE }\end{array}$ & 2014 \\
\hline $\begin{array}{l}\text { Romano, Aldo } \\
\text { Passiante, Giu- } \\
\text { seppina } \\
\text { Del Vecchio, } \\
\text { Pasquale } \\
\end{array}$ & $\begin{array}{l}\text { Knowledge-intensive Entrepre- } \\
\text { neurship in Innovation Ecosystems: } \\
\text { towards the conceptual model of a } \\
\text { Regional Entrepreneurial Learning } \\
\text { Center }\end{array}$ & $\begin{array}{l}\text { 9th International Forum on Knowl- } \\
\text { edge Asset Dynamics }\end{array}$ & 2014 \\
\hline
\end{tabular}

Fonte: Elaborado pelos autores (2017).

No caso das referências de eventos, chama a atenção que esses debates que ocorreram em torno do tema foram realizados em diferentes ocasiões e foram promovidos por diversos autores que até então não apareciam na literatura direcionada à AE. Isso ampliou a propagação da temática e levou o campo de pesquisa da $\mathrm{AE}$ a crescer ainda mais. 
Além dos materiais em capítulos de livros e em eventos, destacou-se naquela ocasião a produção de artigos publicados em diferentes periódicos, não só por aqueles autores já conhecidos (PITTAWAY et al., 2009; RAE; GEE; MOON, 2009; COPE, 2011; PITTAWAY et al., 2011; PITTAWAY; THORPE, 2012; RAE, 2013), mas especialmente por autores sem um histórico de envolvimento com o tema, conforme aponta o quadro a seguir.

Quadro 3 - Referências de artigos em periódicos da terceira fase da AE

\begin{tabular}{|c|c|c|c|}
\hline Autor(es) & Título & Periódico & Ano \\
\hline $\begin{array}{l}\text { Holcomb, Tim R. } \\
\text { et al. }\end{array}$ & $\begin{array}{l}\text { Architecture of entrepreneurial learn- } \\
\text { ing: exploring the link among heuristics, } \\
\text { knowledge, and action }\end{array}$ & $\begin{array}{l}\text { Entrepreneurship } \\
\text { Theory and Practice }\end{array}$ & 2009 \\
\hline $\begin{array}{l}\text { Pihie, Zaidato et } \\
\text { al. }\end{array}$ & $\begin{array}{l}\text { Learning style of university students: Im- } \\
\text { plications for improving entrepreneurial } \\
\text { learning paradigm }\end{array}$ & $\begin{array}{l}\text { International Journal of } \\
\text { Interdisciplinary Social } \\
\text { Sciences }\end{array}$ & 2009 \\
\hline $\begin{array}{l}\text { Ettl, Kerstin } \\
\text { Welter, Friederike }\end{array}$ & $\begin{array}{c}\text { Gender, context and entrepreneurial } \\
\text { learning }\end{array}$ & $\begin{array}{l}\text { International Journal of } \\
\text { Gender and Entrepre- } \\
\text { neurship }\end{array}$ & 2010 \\
\hline $\begin{array}{l}\text { Jiao, Hao } \\
\text { Ogilvie, Dt } \\
\text { Cui, Yu }\end{array}$ & $\begin{array}{c}\text { An empirical study of mechanisms to } \\
\text { enhance entrepreneurs' capabilities } \\
\text { through entrepreneurial learning in an } \\
\text { emerging market }\end{array}$ & $\begin{array}{l}\text { Journal of Chinese En- } \\
\text { trepreneurship }\end{array}$ & 2010 \\
\hline Kuuluvainen, Arto & $\begin{array}{l}\text { Serial entrepreneur and entrepreneurial } \\
\text { learning - a case study from Finland }\end{array}$ & $\begin{array}{l}\text { International Journal of } \\
\text { Business and Globali- } \\
\text { sation }\end{array}$ & 2010 \\
\hline Mckeown, Ian & $\begin{array}{l}\text { No More Heroes: Entrepreneurial Learn- } \\
\text { ing in the SME management team }\end{array}$ & $\begin{array}{l}\text { Industry and Higher } \\
\text { Education }\end{array}$ & 2010 \\
\hline Dave, Crick & $\begin{array}{l}\text { Enterprising individuals and entrepre- } \\
\text { neurial learning: A longitudinal case his- } \\
\text { tory in the UK tourism sector }\end{array}$ & $\begin{array}{l}\text { International Journal } \\
\text { of Entrepreneurial Be- } \\
\text { haviour \& Research }\end{array}$ & 2011 \\
\hline Hamilton, Eleanor & $\begin{array}{l}\text { Entrepreneurial learning in family busi- } \\
\text { ness: A situated learning perspective }\end{array}$ & $\begin{array}{l}\text { Journal of Small Busi- } \\
\text { ness and Enterprise } \\
\text { Development }\end{array}$ & 2011 \\
\hline $\begin{array}{l}\text { Karataş-Özkan, } \\
\text { Mine }\end{array}$ & $\begin{array}{c}\text { Understanding relational qualities of } \\
\text { entrepreneurial learning: Towards a } \\
\text { multi-layered approach }\end{array}$ & $\begin{array}{l}\text { Entrepreneurship \& } \\
\text { Regional Development }\end{array}$ & 2011 \\
\hline $\begin{array}{l}\text { Lagrosen, Stefan } \\
\text { Josefsson, Pernilla }\end{array}$ & $\begin{array}{c}\text { Social media marketing as an entrepre- } \\
\text { neurial learning process }\end{array}$ & $\begin{array}{l}\text { International Journal of } \\
\text { Technology Marketing }\end{array}$ & 2011 \\
\hline $\begin{array}{l}\text { Breslin, Dermot } \\
\text { Jones, Colin }\end{array}$ & $\begin{array}{l}\text { The evolution of entrepreneurial learn- } \\
\text { ing }\end{array}$ & $\begin{array}{l}\text { International Journal of } \\
\text { Organizational Analysis }\end{array}$ & 2012 \\
\hline $\begin{array}{l}\text { De Massis, Alfredo } \\
\text { Minola, Tommaso } \\
\text { Viviani, Diego }\end{array}$ & $\begin{array}{l}\text { Entrepreneurial learning in Italian high- } \\
\text { tech start-ups: An exploratory study }\end{array}$ & $\begin{array}{l}\text { International Journal of } \\
\text { Innovation and Learn- } \\
\text { ing }\end{array}$ & 2012 \\
\hline $\begin{array}{l}\text { Karajic, Dragica } \\
\text { Dabic, Marina } \\
\text { Cingula, Marijan }\end{array}$ & $\begin{array}{c}\text { Interaction among national experts for } \\
\text { the Entrepreneurial Learning, within } \\
\text { pan-European Network }\end{array}$ & $\begin{array}{l}\text { Procedia - Social and } \\
\text { Behavioral Sciences }\end{array}$ & 2012 \\
\hline $\begin{array}{l}\text { Kwong, Caleb C. Y. } \\
\text { et al. }\end{array}$ & $\begin{array}{c}\text { The role of environment in fostering } \\
\text { conductive entrepreneurial learning: } \\
\text { Teaching the 'art' of entrepreneurship in } \\
\text { boot camps }\end{array}$ & $\begin{array}{c}\text { Journal of General Ma- } \\
\text { nagement }\end{array}$ & 2012 \\
\hline
\end{tabular}




\begin{tabular}{|c|c|c|c|}
\hline $\begin{array}{l}\text { Man, Thomas } \\
\text { Wing Yan }\end{array}$ & $\begin{array}{l}\text { Developing a behaviour-centred model } \\
\text { of entrepreneurial learning }\end{array}$ & $\begin{array}{l}\text { Journal of Small Busi- } \\
\text { ness and Enterprise } \\
\text { Development }\end{array}$ & 2012 \\
\hline $\begin{array}{l}\text { Tragazikis, Pana- } \\
\text { giotis } \\
\text { Kirginas, Sotiris } \\
\text { Gouscos, Dimitris }\end{array}$ & $\begin{array}{l}\text { Digital games for entrepreneurial learn- } \\
\text { ing, innovation and creativity: examples } \\
\text { and evaluation criteria }\end{array}$ & $\begin{array}{l}\text { International Journal of } \\
\text { Innovation and Region- } \\
\text { al Development }\end{array}$ & 2012 \\
\hline $\begin{array}{l}\text { Agbim, Kenneth } \\
\text { et al. }\end{array}$ & $\begin{array}{c}\text { Entrepreneurship Development and Tacit } \\
\text { Knowledge: Exploring the Link between } \\
\text { Entrepreneurial Learning and Individual } \\
\text { Know-How }\end{array}$ & $\begin{array}{l}\text { Journal of Business } \\
\text { Studies Quarterly }\end{array}$ & 2013 \\
\hline Chien-Chi, Tseng & $\begin{array}{c}\text { Connecting self-directed learning with } \\
\text { entrepreneurial learning to entrepre- } \\
\text { neurial performance }\end{array}$ & $\begin{array}{l}\text { International Journal } \\
\text { of Entrepreneurial Be- } \\
\text { haviour \& Research }\end{array}$ & 2013 \\
\hline $\begin{array}{l}\text { Owusu-Manu, D. } \\
\text { et al. }\end{array}$ & $\begin{array}{c}\text { Redefining Entrepreneurial Learning Par- } \\
\text { adigms in Developing Countries: A Case } \\
\text { Study of Ghana }\end{array}$ & $\begin{array}{l}\text { Industry and Higher } \\
\text { Education }\end{array}$ & 2013 \\
\hline $\begin{array}{l}\text { Seuneke, Pieter } \\
\text { Lans, Thomas } \\
\text { Wiskerke, Jo- } \\
\text { hannes S. C. }\end{array}$ & $\begin{array}{l}\text { Moving beyond entrepreneurial skills: } \\
\text { Key factors driving entrepreneurial learn- } \\
\text { ing in multifunctional agriculture }\end{array}$ & Journal of Rural Studies & 2013 \\
\hline $\begin{array}{l}\text { Dimitratos, Pavlos } \\
\text { et al. }\end{array}$ & $\begin{array}{l}\text { The overlooked distinction of multina- } \\
\text { tional enterprise subsidiary learning: Its } \\
\text { managerial and entrepreneurial learning } \\
\text { modes }\end{array}$ & $\begin{array}{l}\text { International Business } \\
\text { Review }\end{array}$ & 2014 \\
\hline $\begin{array}{l}\text { Leiva, Juan Carlos } \\
\text { Alegre, Joaquim } \\
\text { Monge, Ricardo }\end{array}$ & $\begin{array}{l}\text { The Influence of Entrepreneurial Learn- } \\
\text { ing in New Firms' Performance: A Study } \\
\text { in Costa Rica }\end{array}$ & Revista Innovar & 2014 \\
\hline $\begin{array}{l}\text { Middleton, Karen } \\
\text { Williams } \\
\text { Donnellon, Anne } \\
\end{array}$ & $\begin{array}{c}\text { Personalizing Entrepreneurial Learning: } \\
\text { A Pedagogy for Facilitating the Know- } \\
\text { Why }\end{array}$ & $\begin{array}{l}\text { Entrepreneurship Re- } \\
\text { search Journal }\end{array}$ & 2014 \\
\hline $\begin{array}{l}\text { Spiteri, Simeon } \\
\text { Maringe, Felix }\end{array}$ & $\begin{array}{l}\text { EU entrepreneurial learning: perspec- } \\
\text { tives of university students }\end{array}$ & $\begin{array}{l}\text { Journal of Enterprising } \\
\text { Communities }\end{array}$ & 2014 \\
\hline $\begin{array}{l}\text { Theodorakopou- } \\
\text { los, Nicholas et al. }\end{array}$ & $\begin{array}{l}\text { Diversifying into technical clothing man- } \\
\text { ufacture as entrepreneurial learning: A } \\
\text { situated learning theory perspective }\end{array}$ & $\begin{array}{c}\text { Journal of Manufactur- } \\
\text { ing Technology Man- } \\
\text { agement }\end{array}$ & 2014 \\
\hline
\end{tabular}

Fonte: Elaborado pelos autores (2017).

$E$, mesmo que houvesse um periódico concentrando a publicação de quatro artigos - Industry and Higher Education, e outras três revistas com dois artigos sobre AE no período - Entrepreneurship and Regional Development; International Journal of Entrepreneurial Behaviour \& Research e Journal of Small Business and Enterprise Development, a maioria dos trabalhos publicados estava disseminada em um número elevado de periódicos.

Dentro dessa fase, o ano de 2014 merece destaque especial pela publicação do levantamento sistemático da literatura entre os temas do "aprender" e do "empreender" realizado por Catherine L. Wang e Harveen Chugh. O artigo intitulado Entrepreneurial Learning: Past Research and Future Challenges (WANG; $\mathrm{CHUGH}, 2014$ ) revelou-se um dos principais materiais para consulta sobre a literatura em $A E$ e, assim como o título remete, apontou para a existência de um futuro de desafios para a temática. Além disso, 
ocorreu nesse ano a publicação de outro livro: Resourcing the start-up business: Creating dynamic entrepreneurial learning capabilities (JONES; MACPHERSON; JAYAWARNA, 2014), que embora não tivesse como foco central a $A E$, trouxe contribuições ao campo do empreendedorismo ao buscar o entendimento do processo de empreender.

Com a descrição desses resultados tem-se o fim do que foi aqui denominada de terceira fase. Na sequência serão apresentados os materiais que foram desenvolvidos a partir do ano de 2015.

\section{A Quarta Fase da AE - o momento atual}

Mais recentemente, apontou-se para o que poderia ser chamada de "quarta fase da Aprendizagem Empreendedora". Isso ocorre não somente pelo fato de que o trabaIho de Wang e Chugh (2014), ao fazer um resgate histórico, indicou um novo horizonte na pesquisa em $A E$, ou pelas discussões que continuavam a ser realizadas em diferentes eventos, conforme apontado no Quadro 4, ou ainda pelo progresso do tema em artigos de revistas, conforme apresentado no Quadro 5.

Quadro 4 - Referências de artigos de eventos da quarta fase da AE

\begin{tabular}{|c|c|c|c|}
\hline Autor(es) & Título & Evento & Ano \\
\hline $\begin{array}{l}\text { Lagrosen, } \\
\text { Stefan }\end{array}$ & $\begin{array}{c}\text { The Role of Entrepreneurial Learning } \\
\text { in Industrial Marketing Success: A } \\
\text { Longitudinal Study }\end{array}$ & $\begin{array}{l}\text { 10th European Conference on Inno- } \\
\text { vation and Entrepreneurship - ECIE }\end{array}$ & 2015 \\
\hline $\begin{array}{l}\text { Lagrosen, } \\
\text { Yvonne } \\
\text { Lagrosen, } \\
\text { Stefan }\end{array}$ & $\begin{array}{l}\text { Entrepreneurial learning for quality } \\
\text { and competitiveness: A study in the } \\
\text { spa-industry }\end{array}$ & $\begin{array}{l}\text { 10th European Conference on Inno- } \\
\text { vation and Entrepreneurship - ECIE }\end{array}$ & 2015 \\
\hline $\begin{array}{l}\text { Platzek, Ber- } \\
\text { nd P. } \\
\text { Pretorius, } \\
\text { Leon }\end{array}$ & $\begin{array}{c}\text { Corporate entrepreneurship educa- } \\
\text { tion: Individual and organizational } \\
\text { entrepreneurial learning }\end{array}$ & $\begin{array}{l}\text { 24th International Association for } \\
\text { Management of Technology - IA- } \\
\text { MOT }\end{array}$ & 2015 \\
\hline $\begin{array}{l}\text { Rusk, Mi- } \\
\text { chele } \\
\text { McGowan, } \\
\text { Pauric }\end{array}$ & $\begin{array}{l}\text { Entrepreneurial Learning in Context: } \\
\text { An Exploration of Learning Models } \\
\text { in Different Domains }\end{array}$ & $\begin{array}{l}\text { 10th European Conference on Inno- } \\
\text { vation and Entrepreneurship - ECIE }\end{array}$ & 2015 \\
\hline $\begin{array}{l}\text { Secundo, } \\
\text { Giustina } \\
\text { et al. }\end{array}$ & $\begin{array}{l}\text { Enhancing corporate entrepreneur- } \\
\text { ship through an entrepreneurial } \\
\text { learning approach: turning students' } \\
\text { ideas into entrepreneurial practice }\end{array}$ & $\begin{array}{l}\text { 10th International Forum on Knowl- } \\
\text { edge Asset Dynamics - IFKAD }\end{array}$ & 2015 \\
\hline $\begin{array}{l}\text { Secundo, } \\
\text { Giustina } \\
\text { et al. }\end{array}$ & $\begin{array}{l}\text { Entrepreneurial learning dynamics } \\
\text { for technology driven entrepreneur- } \\
\text { ship: An integrative framework }\end{array}$ & $\begin{array}{l}\text { 16th European Conference on } \\
\text { Knowledge Management - ECKM }\end{array}$ & 2015 \\
\hline Zheng, Ke & $\begin{array}{l}\text { The Entrepreneurial Learning } \\
\text { Process and Influential Factors of } \\
\text { Entrepreneurs in Business Model } \\
\text { Construction }\end{array}$ & $\begin{array}{c}\text { International Conference on Educa- } \\
\text { tion, Management and Computing } \\
\text { Technology - ICEMCT }\end{array}$ & 2015 \\
\hline $\begin{array}{l}\text { Rossignoli, F. } \\
\text { Lionzo, A. } \\
\text { Lassini, U. }\end{array}$ & $\begin{array}{l}\text { Entrepreneurial learning in family } \\
\text { SMEs: The role of family in commu- } \\
\text { nities of practice }\end{array}$ & $\begin{array}{l}\text { 17th European Conference on } \\
\text { Knowledge Management - ECKM }\end{array}$ & 2016 \\
\hline
\end{tabular}

Fonte: Elaborado pelos autores (2017). 
Quadro 5 - Referências de artigos em periódicos da quarta fase da AE

\begin{tabular}{|c|c|c|c|}
\hline Autor(es) & Título & Periódico & Ano \\
\hline $\begin{array}{l}\text { Clifton, Nick et } \\
\text { al. }\end{array}$ & $\begin{array}{l}\text { An appropriate tool for entrepreneurial } \\
\text { learning in SMEs? The case of the 20Twen- } \\
\text { ty Leadership Programme }\end{array}$ & Local Economy & 2015 \\
\hline $\begin{array}{l}\text { Ernest, Kissi } \\
\text { Matthew, Somi- } \\
\text { ah K. } \\
\text { Samuel, Ansah K. }\end{array}$ & $\begin{array}{c}\text { Towards Entrepreneurial Learning Compe- } \\
\text { tencies: The Perspective of Built Environ- } \\
\text { ment Students }\end{array}$ & Higher Education Studies & 2015 \\
\hline $\begin{array}{l}\text { Hietanen, Lenita } \\
\text { Järvi, Taina }\end{array}$ & $\begin{array}{c}\text { Contextualizing entrepreneurial learning in } \\
\text { basic and vocational education }\end{array}$ & $\begin{array}{l}\text { Journal of Enterprising } \\
\text { Communities }\end{array}$ & 2015 \\
\hline $\begin{array}{l}\text { Hytti, Ulla } \\
\text { Lemmetyinen, } \\
\text { Arja }\end{array}$ & $\begin{array}{l}\text { Social entrepreneurship and entrepreneur- } \\
\text { ial learning in the cultural context }\end{array}$ & $\begin{array}{l}\text { Journal of Enterprising } \\
\text { Communities }\end{array}$ & 2015 \\
\hline $\begin{array}{l}\text { Insulander, Eva } \\
\text { Ehrlin, Anna } \\
\text { Sandberg, Anette }\end{array}$ & $\begin{array}{l}\text { Entrepreneurial learning in Swedish pre- } \\
\text { schools: possibilities for and constraints on } \\
\text { children's active participation }\end{array}$ & $\begin{array}{l}\text { Early Child Development } \\
\text { and Care }\end{array}$ & 2015 \\
\hline $\begin{array}{l}\text { Juhdi, Hasni N. } \\
\text { Hong, Tih Sio } \\
\text { Juhdi, Nurita }\end{array}$ & $\begin{array}{c}\text { Market orientation and entrepreneurial } \\
\text { success: Mediating role of entrepreneurial } \\
\text { learning intensity }\end{array}$ & Journal Pengurusan & 2015 \\
\hline $\begin{array}{l}\text { Baggen, Yvette } \\
\text { et al. }\end{array}$ & $\begin{array}{l}\text { Fostering Entrepreneurial Learning On-the- } \\
\text { Job: evidence from innovative small and } \\
\text { medium-sized companies in Europe }\end{array}$ & $\begin{array}{l}\text { European Journal of Edu- } \\
\text { cation }\end{array}$ & 2016 \\
\hline $\begin{array}{l}\text { El Hallam, Horia } \\
\text { St-Jean, Étienne }\end{array}$ & $\begin{array}{l}\text { Nurturing Entrepreneurial Learning } \\
\text { through Mentoring }\end{array}$ & $\begin{array}{l}\text { Journal of Developmental } \\
\text { Entrepreneurship }\end{array}$ & 2016 \\
\hline $\begin{array}{l}\text { Muhe, Arniati } \\
\text { Tawe, Aamiru- } \\
\text { ddin }\end{array}$ & $\begin{array}{l}\text { The effect of the entrepreneurial learn- } \\
\text { ing design on students' entrepreneurial } \\
\text { competence in vocational high schools in } \\
\text { Makassar }\end{array}$ & $\begin{array}{l}\text { International Journal of } \\
\text { Environmental and Sci- } \\
\quad \text { ence Education }\end{array}$ & 2016 \\
\hline $\begin{array}{l}\text { Ramsgaard, Mi- } \\
\text { chael Breum } \\
\text { Christensen, } \\
\text { Marie Ernest }\end{array}$ & $\begin{array}{c}\text { Interplay of entrepreneurial learning } \\
\text { forms: a case study of experiential learning } \\
\text { settings }\end{array}$ & $\begin{array}{l}\text { Innovations in Education } \\
\text { and Teaching Interna- } \\
\text { tional }\end{array}$ & 2016 \\
\hline $\begin{array}{l}\text { Saluto, Paolo } \\
\text { Guelfi, Silvano } \\
\text { Norese, Maria } \\
\text { Franca }\end{array}$ & $\begin{array}{l}\text { An integration of theoretical knowledge, } \\
\text { day by day experience and multicriteria } \\
\text { methods to support the entrepreneurial } \\
\text { learning process }\end{array}$ & $\begin{array}{l}\text { Measuring Business Ex- } \\
\text { cellence }\end{array}$ & 2016 \\
\hline $\begin{array}{l}\text { Täks, Marge } \\
\text { Tynjälä, Päivi } \\
\text { Kukemelk, Hasso }\end{array}$ & $\begin{array}{l}\text { Engineering students' conceptions of } \\
\text { entrepreneurial learning as part of their } \\
\text { education }\end{array}$ & $\begin{array}{l}\text { European Journal of Engi- } \\
\text { neering Education }\end{array}$ & 2016 \\
\hline
\end{tabular}

Fonte: Elaborado pelos autores (2017).

Esse novo horizonte na pesquisa em $\mathrm{AE}$, no entanto, surgiu principalmente pela publicação do livro Entrepreneurial Learning: New perspectives in research, education and practice (RAE; WANG, 2015). Essa obra, dedicada exclusivamente à Aprendizagem Empreendedora, surge com a pretensão de orientar o campo acadêmico para as novas possiblidades de pesquisa. David Rae e Catharine L. Wang, assim como fizeram Richard Harrison e Claire Leitch anos antes, organizaram um livro em que são envolvidos diversos autores, os quais revisam o campo sobre o tema e alguns ainda propõem um avanço no que se refere ao desenvolvimento de conhecimento sobre o assunto, ao sugerirem 
que se estabeleça uma nova orientação nas pesquisas sobre Aprendizagem Empreendedora. Isto é feito por meio de trabalhos empíricos que utilizaram, além dos fundamentos teóricos já existentes, novas sugestões para a observação desse fenômeno. Essas recomendações sugerem ir além de uma visão cognitiva e experiencial desse tipo de aprendizagem. Assim, o livro apresenta-se como uma revisão na literatura sobre Aprendizagem Empreendedora, oferecendo o esclarecimento dessas duas, mas também de outras diferentes e complementares perspectivas existentes, abrindo assim possibilidades para o futuro desenvolvimento da temática.

Após os anos de 2015 e 2016 serem marcados pelo que acaba de ser relatado, questiona-se: E o ano de 2017? Como tem sido esse período até o momento? Uma rápida resposta a essa pergunta poderia ser: Promissor! Já houve um número considerável de publicações sobre o tema, seja em capítulos de livros (GONSALVES; ZAMORA, 2017; JÄMINKI, 2017; JONES; NEWBERY; UNDERWOOD, 2017; SECUNDO et al., 2017), ou então em periódicos (DEMETI; SULJOTI; DEMETI, 2017; KUBBERØD; PETTERSEN, 2017), mas essa resposta decorre particularmente pela edição especial da revista International Journal of Entrepreneurial Behaviour and Research - Entrepreneurial learning dynamics in knowledge-intensive enterprises, em que vários artigos sobre o tema foram publicados, conforme quadro a seguir.

Quadro 6 - Artigos da edição especial da revista International Journal of Entrepreneurial Behaviour and Research - 2017

\begin{tabular}{|c|c|}
\hline Autor(es) & Título \\
\hline $\begin{array}{l}\text { Cannavacciuolo, Lorella } \\
\text { landoli, Luca } \\
\text { Ponsiglione, Cristina } \\
\text { Zollo, Giuseppe }\end{array}$ & $\begin{array}{l}\text { Learning by failure vs learning by habits: Entrepreneurial learning mi- } \\
\text { cro-strategies as determinants of the emergence of co-located entre- } \\
\text { preneurial networks }\end{array}$ \\
\hline $\begin{array}{l}\text { Cantino, Valter } \\
\text { Devalle, Alain } \\
\text { Cortese, Damiano } \\
\text { Ricciardi, Francesca } \\
\text { Longo, Mariangela }\end{array}$ & $\begin{array}{l}\text { Place-based network organizations and embedded entrepreneurial } \\
\text { learning: Emerging paths to sustainability }\end{array}$ \\
\hline $\begin{array}{l}\text { El-Awad, Ziad } \\
\text { Gabrielsson, Jonas } \\
\text { Politis, Diamanto }\end{array}$ & $\begin{array}{l}\text { Entrepreneurial learning and innovation: the critical role of team } \\
\text { level learning for the evolution of innovation capabilities in technolo- } \\
\text { gy-based ventures }\end{array}$ \\
\hline Rae, David & Entrepreneurial learning: peripherality and connectedness \\
\hline $\begin{array}{l}\text { Scarmozzino, Emanuela } \\
\text { Corvello, Vincenzo } \\
\text { Grimaldi, Michele }\end{array}$ & $\begin{array}{l}\text { Entrepreneurial learning through online social networking in high-tech } \\
\text { startups }\end{array}$ \\
\hline $\begin{array}{l}\text { Secundo, Giustina } \\
\text { Del Vecchio, Pasquale } \\
\text { Schiuma, Giovanni } \\
\text { Passiante, Giuseppina }\end{array}$ & $\begin{array}{l}\text { Activating entrepreneurial learning processes for transforming univer- } \\
\text { sity students' idea into entrepreneurial practices }\end{array}$ \\
\hline $\begin{array}{l}\text { Secundo, Giustina } \\
\text { Schiuma, Giovanni } \\
\text { Passiante, Giuseppina }\end{array}$ & Entrepreneurial learning dynamics in knowledge-intensive enterprises \\
\hline Soetanto, Danny & Networks and entrepreneurial learning: coping with difficulties \\
\hline $\begin{array}{l}\text { Walsh, Grace S. } \\
\text { Cunningham, James A. }\end{array}$ & $\begin{array}{l}\text { Regenerative failure and attribution: Examining the underlying process- } \\
\text { es affecting entrepreneurial learning }\end{array}$ \\
\hline
\end{tabular}

Fonte: Elaborado pelos autores (2017). 
Assim, diante do exposto, uma vez realizada a observação histórica do desenvolvimento da $A E$ e, à medida que se afirma que "depois de 20 anos, a Aprendizagem Empreendedora não pode ser considerada como um novo ou emergente campo de estudo, mas como um campo que se estabeleceu e tem sido subdividido em um número relativo de tópicos relacionados" (RAE; WANG, 2015, p. 8), torna-se necessário um olhar sobre os diferentes enfoques utilizados na construção desse tema no decorrer dos anos, para que primeiramente seja possível identificar essa trajetória e em um segundo momento também seja possível enxergar o que pode estar por vir no que diz respeito à mudança na observação e estudo desse fenômeno.

Nesse sentido, uma vez apresentadas as diferentes fases em que a aprendizagem empreendedora teve o seu desenvolvimento na condição de campo de estudo, surge o interesse de realizar uma observação mais profunda nas diferentes perspectivas (e seus pressupostos) utilizadas para a pesquisa endereçada à $A E$, a fim de se ter uma compreensão mais específica do tema. Assim, na próxima seção serão expostos os aspectos que dizem respeito a essa questão.

\section{AE: do surgimento em direção às novas possibilidades de estudo}

Após o aparecimento desse campo de estudo baseado na literatura em empreendedorismo e em Aprendizagem Organizacional (HARRISON; LEITCH, 2008; ERDÉLYI, 2010), entre as perspectivas em $A E$ que surgiram destacam-se a cognitivista e a experiencial (YOUNG; SEXTON, 1997; RAE, 2000, 2005a; AGBIM; OWUTUAMOR; ORIAREWO, 2013), ambas com ênfase em aspectos individuais. Mais recentemente surgiram discussões que retiram o foco no indivíduo a fim de dar maior centralidade ao coletivo e ao social (PITTAWAY; COPE, 2007; HARRISON; LEITCH, 2008; MCHENRY, 2008; MCKEOWN, 2015; RAE; WANG, 2015). Cada uma dessas perspectivas é empregada dentro de subtemas que compõem a $A E$, como a educação empreendedora, as competências empreendedoras, a orientação empreendedora e o reconhecimento de oportunidades (RAE; CARSWELL, 2000; 2001; LÖBLER, 2006; BLENKER et al., 2012; ZAMPIER; TAKAHASHI, 2014; RAE; WANG, 2015; BAGGEN et al., 2016; DIAS; MARTENS, 2016).

Ou seja, existe uma gama de possibilidades que o guarda-chuva da Aprendizagem Empreendedora como tema abrange. Dessa maneira, uma vez caracterizada a forma como foram conduzidas até então as pesquisas dentro do tema da $A E$ e em virtude da expectativa gerada com a publicação dos trabalhos mais recentes (WANG; CHUGH, 2014, 2015; RAE; WANG, 2015), que sugerem novas perspectivas, as quais recomendam que se afaste da visão individual - cognitiva e experiencial - espera-se que surjam novas pesquisas que empreguem diferentes perspectivas das até então utilizadas.

No cenário nacional, a necessidade de avançar na produção de conhecimento sobre AE decorre do fato de que só a partir de 2010 passou-se a utilizar a literatura internacional disponível sobre o tema em pesquisas no Brasil, além do que, atualmente, ao serem realizadas pesquisas sobre $A E$, utiliza-se predominantemente como base teórica as perspectivas até então hegemônicas (cognitiva e experiencial), sem apresentar nenhuma novidade substancial no que se refere ao avanço teórico dentro da temática. Isso fica evidente à medida que se analisa a divulgação da primeira dissertação de Mestrado sobre o tema de Zampier (2010), que resultou um ano depois em uma publicação em um 
periódico (ZAMPIER; TAKAHASHI, 2011), bem como a segunda dissertação encontrada na literatura nacional dedicada à AE defendida por Fiala (2012), ano marcado também pela aparição de outros materiais sobre o tema (GOIS; MACHADO, 2012; ZAMPIER; TAKAHASHI, 2012).

Já a terceira dissertação surgiu no ano seguinte (SACRAMENTO, 2013), assim como mais alguns artigos que foram apresentados em eventos nesse período e também no ano seguinte (DA SILVA; TEIXEIRA, 2013; DIAS; MARTENS, 2014; MINELLO; SCHERER; RAMOS, 2014). O ano de 2014 é marcado pelo aparecimento da publicação de outro artigo da autora que foi quem desenvolveu primeiramente o tema no cenário nacional por meio de uma dissertação quatro anos antes (ZAMPIER; TAKAHASHI, 2014) e também pela publicação de mais um trabalho fruto de pesquisa de Mestrado (SILVA, 2014). Ambos os trabalhos caracterizam-se basicamente pelo emprego de bases teóricas das fases iniciais da aprendizagem.

Assim, uma vez que é reconhecida a necessidade de mudanças, especialmente em direção a um avanço das formas de se estudar o processo de aprendizagem dos empreendedores, entre as novas possibilidades destaca-se a visão social da aprendizagem. Principalmente com o emprego das teorias da prática que veem o mundo como um conjunto contínuo, com nexos e de alianças de práticas (NICOLINI, 2012) e que consideram a prática como o locus do aprendizado (GHERARDI, 2006, 2009; GHERARDI; STRATI, 2014) e fonte de conhecimento. Essa abordagem revela-se promissora na medida em que tem como uma de suas intenções complementar (e superar) a visão cognitiva e experiencial da Aprendizagem Empreendedora.

Desse modo, após essa revisão sistemática no campo de estudo e um levantamento minucioso da literatura nacional conforme apresentado no decorrer dessa pesquisa, percebe-se quão jovem é o estudo desse tema e como existe uma lacuna aberta para a realização de futuros trabalhos. Isso deve ocorrer não somente com a utilização de metodologias diferentes, mas com o emprego de enfoques que vão além do ensino do empreendedorismo (DOLABELA; FILION, 2013) ou da formação de competências empreendedoras (ZAMPIER; TAKAHASHI, 2014; LEITE; DIAS, 2015; DIAS; MARTENS, 2016), mas na compreensão de como o processo da aprendizagem ocorre em empreendedores.

O destaque na natureza situada da Aprendizagem Empreendedora é visto nesse sentido como uma possibilidade para futuros estudos, seguindo o que apontam as pesquisas com esse escopo no cenário internacional, como Terzieva (2016) e Rossignoli, Lionzo e Lassini (2016), que exploraram o conceito de comunidades de práticas na relação com à AE. Ou então, Hafeez et al. (2018), que recentemente investigaram o uso das redes sociais pelos empreendedores para interagir e compartilhar experiências. Nesse sentido, considera-se a $A E$ como um processo que ocorre nas práticas diárias. Como apontado por Cannavacciuolo et al. (2017) ao analisar o impacto de práticas na aprendizagem, assumindo que a interação social no ambiente em que os empreendedores operam agrega conhecimento. De forma semelhante, Kubberød e Pettersen (2017) observaram a geração de conhecimento por meio de um aprendizado que ocorre em uma experiência social. Secundo et al. (2017) também defendem essa ideia ao relataram que alunos que se integraram a diversos atores (sociais) por meio de atividades geram aprendizado e, consequentemente, algum tipo de resultado - seja esse um produto, uma inovação ou, até mesmo, uma nova organização. Ou seja, em ambos os casos exis- 
te a consideração de que a aprendizagem empreendedora possui uma conexão com as práticas sociais em que os indivíduos se envolvem, seja na esfera pessoal ou professional, seja na educação formal ou na busca de conhecimento no dia a dia.

\section{CONSIDERAÇÕES FINAIS}

Após realizado o levantamento sistemático da literatura sobre AE nos âmbitos internacional e nacional com o emprego de critérios definidos, que se pautaram na busca de uma amplitude de diferentes fontes de pesquisa, com a utilização de recursos de software a fim de dar maior credibilidade no processo de controle dos resultados, ainda assim pode-se considerar que essa pesquisa tem suas limitações. Por isso, reconhecemos que podem haver (e existem de fato) mais estudos na literatura nacional e internacional que dizem respeito à Aprendizagem Empreendedora. A intenção dos autores não é a de afirmar que todo o campo foi mapeado. Isso fica evidente quando observado que alguns trabalhos sobre a $A E$ não apareceram nos resultados da pesquisa, mas foram identificados nas referências bibliográficas de materiais analisados no decorrer do levantamento.

Assim, enfatizam-se trabalhos de Jason Cope que aparece na lista de autores com a maior quantidade de publicações sobre a temática, e que principalmente com alguns colaboradores possui outros artigos desenvolvidos para eventos (COPE; HAMILTON, 2005; COPE; PITTAWAY, 2005; COPE, 2008; COPE; CAVE; ECCLES, 2008). Também foi identificado um trabalho de David Rae (RAE, 2006), entre outros estudos de autores diversos (MURPHY, 1993; YOUNG; SEXTON, 1997; PLATZEK; PRETORIUS; WINZKER, 2014; JÄMINKI, 2016). Existem referências na literatura nacional não citadas, com destaque para a dissertação de José Luis Gonçalves Ramos (RAMOS, 2015), que resultou em um dos artigos que apareceu na pesquisa (MINELLO; SCHERER; RAMOS, 2015) e o artigo de Moraes e Hoeltgebaum (2003) apresentado no Third International Conference of the Iberoamerican Academy of Management, que é considerado por Zampier e Takahashi (2014, p. 5) como sendo o primeiro "modelo para investigação e análise do processo de aprendizagem empreendedora".

Posto isso, convém também frisar que a elaboração de um levantamento sistemático da literatura não é apenas uma investigação sobre o quem, o quando e o onde o tema em questão foi (e tem sido) explorado, mas essa tarefa também deve ser encarada como uma possibilidade que pode proporcionar o entendimento do "o que" e do "como" os estudos têm sido conduzidos. E nesta pesquisa não foi diferente. Desde o surgimento da Aprendizagem Empreendedora como campo de estudo de forma mais intensa no final dos anos 90 até as pesquisas mais recentes, apontaram-se as mudanças que ocorreram e foram destacadas as diferentes abordagens que têm sido utilizadas.

Diante do exposto, este artigo não deseja ser apenas um levantamento da literatura, mas tem como intenção contribuir para o avanço do campo da $A E$ na medida em que revela a incipiência desse campo, especialmente na literatura nacional. Ou seja, após a pesquisa mapear o campo de estudo da $\mathrm{AE}$, ela não olha somente para o passado, mas também visa-se a apontar para o futuro a fim de orientar e sugerir novas possibilidades de realização de pesquisas empíricas. Dessa forma, salienta-se que o estudo sobre a $A E$ é um processo inacabado, por isso sugere-se que se busque avançar na compreensão do processo de aprendizagem dos empreendedores por meio de investigações que em- 
preguem diferentes abordagens. Isso deve ocorrer de forma mais acentuada especialmente na literatura nacional, devido ao baixo número de publicações e à forma como elas têm sido conduzidas até o momento.

Para isso propõe-se que sejam utilizadas abordagens que enfoquem os aspectos sociais da aprendizagem, de forma diferente da perspectiva cognitiva e experiencial. Assim, recomenda-se que sejam utilizadas as teorias da prática conforme apresentam Gherardi (2006), Gherardi e Strati (2014) e Nicolini (2012) e, mais recentemente Nicolini (2016) e Nicolini e Monteiro (2017) nos estudos empíricos sobre Aprendizagem Empreendedora. Isso implica, por exemplo, beneficiar-se da visão das comunidades de prática de Wenger (1998) e da aprendizagem situada de Lave e Wenger (1991) como considerado por Terzieva (2016) e Rae (2017). Ou seja, sugere-se que a aprendizagem empreendedora seja concebida como um fenômeno complexo e que se leve em consideração diferentes aspectos que compõem esse processo: individuais, sociais, ambientais e também históricos. Isso envolve a busca pelo entendimento da Aprendizagem Empreendedora como um processo que decorre mesmo antes de a própria ação empreendedora ser realizada, na medida que os indivíduos se envolvem em diferentes práticas sociais.

Por fim, destaca-se que o objetivo de mapear o campo sobre a Aprendizagem Empreendedora foi alcançado e que foi apresentada, de forma sistemática, a literatura base para a produção de futuros trabalhos que visem a essa temática como foco de investigação. A realização de estudos com a intenção de um entendimento mais profundo da Aprendizagem Empreendedora como um processo social e firmado nas práticas trará contribuições não só para a teorização sobre o assunto, mas também para que os atuais e futuros empreendedores possam ter conhecimento sobre esse fenômeno no qual eles se envolvem ou então farão parte. Principalmente pelo fato de que o entendimento da Aprendizagem Empreendedora enquanto fenômeno está diretamente ligado ao empreendedorismo e à gestão de empresas. Ou seja, a aprendizagem empreendedora pode proporcionar maiores condições do desenvolvimento de novos negócios, bem como a aplicação das competências empreendedoras na gestão empresarial.

\section{REFERÊNCIAS}

AGBIM, K. C.; OWUTUAMOR, Z. B.; ORIAREWO, G. O. Entrepreneurship Development and Tacit Knowledge: Exploring the Link between Entrepreneurial Learning and Individual Know-How. Journal of Business Studies Quarterly, v. 5, n. 2, p. 112-129, 2013. ISSN 21521034.

AKRITIDIS, L.; KAKOURIS, A. Modelling the Style in Entrepreneurial Learning From Experience. EUROPEAN CONFERENCE ON INNOVATION AND ENTREPRENEURSHIP - ECIE, 7., 2012. Santarém: Academic Publishing International, 2012. p. 1-7.

BAGGEN, Y. et al. Fostering Entrepreneurial Learning On-the-Job: evidence from innovative small and medium-sized companies in Europe. European Journal of Education, v. 51, n. 2, p. 193-209, 2016. ISSN 01418211.

BAUMOL, W. J. Entrepreneurship in economic theory. Annual Meeting of the American Economic Association, Chicago, n. 58, p. 64-71, 1968.

BERGLUND, H.; HELLSTRÖM, T.; SJÖLANDER, S. Entrepreneurial learning and the role of venture capitalists. Venture Capital, v. 9, n. 3, p. 165-181, 2007. ISSN 13691066.

BLENKER, P. et al. Entrepreneurship as everyday practice: towards a personalized pedagogy of enterprise education. Industry and Higher Education, v. 26, n. 6, p. 417-430, 2012. ISSN 09504222.

BRESLIN, D.; JONES, C. The evolution of entrepreneurial learning. International Journal of Organizational Analysis, v. 20, n. 3, p. 294-308, 2012. ISSN 1934-8835. 
BREUER, H.; MAHDJOUR, S. Lean Venturing: Entrepreneurial Learning to Model and Grow New Business. INNOVATION SYMPOSIUM - STIMULATING INNOVATION: CHALLENGES FOR MANAGEMENT, SCIENCE \& TECHNOLOGY, 5., 2012. Seoul: The International Society for Professional Innovation Management, 2012. p. 1-14.

BYGRAVE, W.; MINNITI, M. The social dynamics of entrepreneurship. Entrepreneurship Theory and Practice, v. 24, n. 3, p. 25-36, 2000. ISSN 1042-2587.

BYGRAVE, W. D. The Entrepreneurial Process. In: BYGRAVE, W. D.; ZACHARAKIS, A. (ed.). The Portable MBA in Entrepreneurship. 4 th. ed. Hoboken, Nova Jersey, EUA: John Wiley \& Sons, Inc., 2009. p. 1-26. Cap. 1. ISBN 9781118256121.

CAMPOS, H. M.; PARELLADA, F. S.; PALMA, Y. Mapping the Intellectual Structure of Entrepreneurship Research: revisiting the invisible college. Revista Brasileira de Gestão de Negócios, v. 14, n. 42, p. 41-58, 2012. ISSN 1806-4892.

CANNAVACCIUOLO, L. et al. Learning by failure vs learning by habits: Entrepreneurial learning micro-strategies as determinants of the emergence of co-located entrepreneurial networks. International Journal of Entrepreneurial Behaviour and Research, v. 23, n. 3, p. 524-546, 2017. ISSN 13552554.

CANTINO, V. et al. Place-based network organizations and embedded entrepreneurial learning: Emerging paths to sustainability. International Journal of Entrepreneurial Behaviour and Research, v. 23, n. 3, p. 504-523, 2017. ISSN 13552554.

CHEN, W.-T.; LI, X.-C. Entrepreneurial Learning in New Ventures: Dimensions Developing and Structure Testing. International Conference on Management Science \& Engineering, 2009, Moscow. p. 1.677-1.684. CHIEN-CHI, T. Connecting self-directed learning with entrepreneurial learning to entrepreneurial performance. International Journal of Entrepreneurial Behaviour \& Research, v. 19, n. 4, p. 425-446, 2013. ISSN 1355-2554.

$\mathrm{CHOU}, \mathrm{C}$. M. et al. The influencing factors of student teachers' entrepreneurial learning behavior. INTERNATIONAL JOINT CONFERENCE ON COMPUTATIONAL SCIENCES AND OPTIMIZATION - CSO, 4., 2011. Kunming/Lijiang, 2011. p. 531-534.

CLIFTON, N. et al. An appropriate tool for entrepreneurial learning in SMEs? The case of the 20Twenty Leadership Programme. Local Economy, v. 30, n. 5, p. 534-556, 2015. ISSN 0269-0942.

COPE, J. Entrepreneurial learning and critical reflection: Discontinuous events as triggers for "higher-level" learning. Management Learning, v. 34, n. 4, p. 429-450, 2003. ISSN 1350-5076.

COPE, J. Toward a dynamic learning perspective of entrepreneurship. Entrepreneurship Theory and Practice, v. 29, n. 4, p. 373-397, 2005.

COPE, J. Same, same but different: confronting the entrepreneurial learning task through critical episodes. Glasgow: Scottish Entrepreneurship Seminar, 2008.

COPE, J. Entrepreneurial learning from failure: An interpretative phenomenological analysis. Journal of Business Venturing, v. 26, n. 6, p. 604-623, 2011. ISSN 0883-9026.

COPE, J.; CAVE, F.; ECCLES, S. The Impact and Outcomes of Venture Failure: An Entrepreneurial Learning Perspective In: CENTER, B. C. Frontiers of Entrepreneurship Research, Babson Park, 2008, p. 4.

COPE, J.; HAMILTON, E. Toward a theory of socially situated entrepreneurial learning. Oxford: British Academy of Management Conference, 2005.

COPE, J.; PITTAWAY, L. Applying an entrepreneurial learning lens to entrepreneurship education. Oxford: British Academy of Management Conference, 2005.

COPE, J.; WATTS, G. Learning by doing - An exploration of experience, critical incidents and reflection in entrepreneurial learning. International Journal of Entrepreneurial Behaviour \& Research, v. 6, n. 3, p. 104124, 2000. ISSN 1355-2554.

DA SILVA, J. D.; TEIXEIRA, R. M. Aprendizagem empreendedora: um estudo de casos múltiplos com empreendedores sociais de Aracaju - Sergipe. SEMEAD, 16., 2013. São Paulo 2013.

DAVE, C. Enterprising individuals and entrepreneurial learning: A longitudinal case history in the UK tourism sector. International Journal of Entrepreneurial Behaviour \& Research, v. 17, n. 2, p. 203-218, 2011. ISSN 1355-2554.

DEAKINS, D.; FREEL, M. Entrepreneurial learning and the growth process in SMEs. The Learning Organization, v. 5, n. 3, p. 144-155, 1998. ISSN 0969-6474.

DEAKINS, D.; SULLIVAN, R.; WHITTAM, G. Developing support for entrepreneurial learning: evidence from start-up support programs. International Journal of Entrepreneurship and Innovation Management, v. 2, n. 4-5, p. 323-338, 2002. ISSN 1368275X

DE MASSIS, A.; MINOLA, T.; VIVIANI, D. Entrepreneurial learning in Italian high-tech start-ups: An exploratory study. International Journal of Innovation and Learning, v. 11, n. 1, p. 94-114, 2012. ISSN 14718197 
DEMETI, A.; SULJOTI, E. R.; DEMETI, T. Entrepreneurship and entrepreneurial learning in Albania. World Review of Entrepreneurship, Management and Sustainable Development, v. 13, n. 2-3, p. 252-270, 2017. ISSN 17460573

DENG, Y.-Y.; WANG, S.-S. Research on the role of network in entrepreneurial learning of new venture. WEST LAKE INTERNATIONAL CONFERENCE ON SMALL AND MEDIUM BUSINESS, 9., 2008. Hangzhou, 2008. p. 551-556.

DENG, Y.; WANG, S. The process of entrepreneurial learning in new venture. CONFERENCE ON SYSTEMS SCIENCE, MANAGEMENT SCIENCE AND SYSTEM DYNAMICS, 2007. Shanghai, 2007. p. 343-351.

DENYER, D.; TRANFIELD, D. Producing a systematic review. In: BUCHANAN, D.; BRYMAN, A. (ed.). The Sage Handbook of Organizational Research Methods. London: Sage, 2008. p. 671-689. Cap 39. ISBN 1446200647

DIAS, T. R. F. V.; MARTENS, C. D. P. Aprendizagem empreendedora e conhecimento empreendedor em contexto de insucesso empresarial: proposições preliminares. ENANPAD, 38., 2014. Rio de Janeiro, 2014.

DIAS, T. R. F. V.; MARTENS, C. D. P. Competências e aprendizagem empreendedora no contexto de insucesso empresarial: proposição de um modelo conceitual. Desenvolvimento em Questão, ljuí: Ed. Unijuí, v. 14, n. 33, p. 172-202, 2016. ISSN 2237-6453.

DICKEL, P.; ANDREE, H. Entrepreneurial learning in energy technology start- ups: A case study in the biogas market. In: WÜSTENHAGEN, R.; WUEBKER, R. (ed.). Handbook of Research on Energy Entrepreneurship. Cheltenham, UK: Edward Elgar Publishing, 2011. p. 58-82. Cap. 4. ISBN 9781848445512.

DIMITRATOS, P. et al. The overlooked distinction of multinational enterprise subsidiary learning: Its managerial and entrepreneurial learning modes. International Business Review, v. 23, n. 1, p. 102-114, 2014. ISSN 0969-5931.

DOLABELA, F.; FILION, L. J. Fazendo revolução no Brasil: a introdução da pedagogia empreendedora nos estágios iniciais da educação. Revista de Empreendedorismo e Gestão de Pequenas Empresas - Regepe, v. 3, n. 2, p. 134-181, 2013.

DUFFY, S. G. Entrepreneurial learning: Exploring unexpected key events in the post-startup period. (Ph.D.). Washington: School of Business; George Washington University, 2007. 263p.

EL-AWAD, Z.; GABRIELSSON, J.; POLITIS, D. Entrepreneurial learning and innovation: The critical role of team-level learning for the evolution of innovation capabilities in technology-based ventures. International Journal of Entrepreneurial Behaviour and Research, v. 23, n. 3, p. 381-405, 2017. ISSN 13552554.

EL HALLAM, H.; ST-JEAN, É. Nurturing Entrepreneurial Learning through Mentoring. Journal of Developmental Entrepreneurship, v. 21, n. 2, p. 1-18, 2016. ISSN 1084-9467.

ERDÉLYI, P. The Matter of Entrepreneurial Learning: A Literature Review. INTERNATIONAL CONFERENCE ON ORGANIZATIONAL LEARNING, KNOWLEDGE AND CAPABILITIES - OLKC. Boston: Northeastern University, 2010.

ERIKSON, T. Towards a taxonomy of entrepreneurial learning experiences among potential entrepreneurs. Journal of Small Business and Enterprise Development, v. 10, n. 1, p. 106-112, 2003. ISSN 1462-6004.

ERNEST, K.; MATTHEW, S. K.; SAMUEL, A. K. Towards Entrepreneurial Learning Competencies: The Perspective of Built Environment Students. Higher Education Studies, v. 5, n. 1, p. 20-30, 2015. ISSN 19254741.

ETTL, K.; WELTER, F. Gender, context and entrepreneurial learning. International Journal of Gender and Entrepreneurship, v. 2, n. 2, p. 108-129, 2010. ISSN 1756-6266.

FESTERVAND, T. A.; FORREST, J. E. Entrepreneurial Preparedness: A Multi-Stage Model. Journal of Business and Entrepreneurship, v. 5, n. 3, 1993.

FIALA, N. As incubadoras como instrumento effectual de aprendizagem do empreendedorismo. 2012. 125p. Dissertação (Mestrado) - Escola de Administração de Empresas de São Paulo, Fundação Getúlio Vargas, São Paulo, 2012.

FORBES, D. P. The infrastructure of entrepreneurial learning. In: MITCHELL, R. J.; MITCHELL, R. K. et al. (ed.). Handbook of Entrepreneurial Cognition. Cheltenham: Edward Elgar Publishing, 2014. p. 364-382. Cap. 10. ISBN 9781781006597.

FULFORD, H.; BAILEY, M. Journals and Jottings on Entrepreneurial Learning Journeys. EUROPEAN CONFERENCE ON INNOVATION AND ENTREPRENEURSHIP, 2014. Belfast, 2014. p. 198-206.

GABRIELSSON, J.; POLITIS, D. Entrepreneurial learning and innovation: Building entrepreneurial knowledge from career experience for the creation of new ventures. In: NOBRE, F. S.;WALKER, D. et al. (ed.). Technological, Managerial and Organizational Core Competencies: Dynamic Innovation and Sustainable Development. Hershey: Business Science IGI Global, 2012. p. 245-263. Cap. 14. ISBN 9781613501658. 
GARLICK, R. Entrepreneurial learning in a secure E-Commerce course through creating competitive real-world sites. INTERNATIONAL CONFERENCE ON E-COMMERCE, E-BUSINESS AND E-SERVICE - EEE. Hong Kong, 2014. p. 29-31.

GARTNER, W. B. "Who is an Entrepreneur?" Is the Wrong Question. American Journal of Small Business, v. 12, n. 4, p. 11-32, 1988.

GHERARDI, S. Organizational Knowledge: The Texture of Workplace Learning. Oxford: BlackWell Publishing, 2006. ISBN 1405125594.

GHERARDI, S. Knowing and learning in practice-based studies: an introduction. The Learning Organization, v. 16, n. 5, p. 352-359, 2009. ISSN 0969-6474.

GHERARDI, S.; STRATI, A. Administração e aprendizagem na prática. Rio de Janeiro: Elsevier, 2014. ISBN 978-85-352-7928-3.

GOIS, P. H. D.; MACHADO, H. P. V. Uma abordagem sobre o papel das redes para pequenas empresas e sobre os efeitos no aprendizado de empreendedores. Revista de Empreendedorismo e Gestão de Pequenas Empresas - Regepe, v. 1, n. 1, 2012.

GOMES, V. Empreendedorismo nas organizações que aprendem. Considerações sobre a dicotomia: Ensino versus aprendizado do empreendedorismo. ENCONTRO DE ESTUDOS SOBRE EMPREENDEDORISMO E GESTÃO DE PEQUENAS EMPRESAS - EGEPE, 1., 2000. Maringá, 2000.

GONSALVES, E.; ZAMORA, R. Ludic executives: The case for play in entrepreneurial learning design. In: JONES, P.; MAAS, G. et al. (ed.). Entrepreneurship Education: Contemporary Issues in Entrepreneurship Research. Bingley, Reino Unido: Emerald Group Publishing, 2017. p. 213-233. V. 7. ISBN 978-1-78714-281. GUARDANI, F.; ROMITO, F. o processo de aprendizagem dos empreendedores: uma análise da forma como gestores de micro empresas aprenderam a administrar seus negócios. ENCONTRO DE ESTUDOS SOBRE EMPREENDEDORISMO E GESTÃO DE PEQUENAS EMPRESAS - EGEPE, 5., 2008. São Paulo, 2008.

HADJIELIAS, E.; HAMILTON, E.; HOWORTH, C. Entrepreneurial learning in the family management group: A social organizational learning perspective. In: SMYRNIOS, K. X.; POUTZIOURIS, P. Z. et al. (ed.). Handbook of Research on Family Business. 2th ed. Cheltenham: Edward Elgar Publishing, 2013. p. 451-466. Cap. 20. ISBN 978-1848443228.

HAFEEZ, K. et al. How do entrepreneurs learn and engage in an online community-of-practice? A case study approach. Behaviour \& Information Technology, v. 37, n. 7, p. 714-735, 2018.

HAMILTON, E. Entrepreneurial learning in family business: A situated learning perspective. Journal of Small Business and Enterprise Development, v. 18, n. 1, p. 8-26, 2011. ISSN 1462-6004.

HANNON, P. Entrepreneurial Learning and Mentoring. International Small Business Journal, v. 19, n. 2, p. 100, 2001. ISSN 0266-2426.

HARRISON, R. T.; LEITCH, C. M. Entrepreneurial learning: Researching the interface between learning and the entrepreneurial context. Entrepreneurship Theory and Practice, v. 29, n. 4, p. 351-371, 2005. ISSN 1042-2587.

HARRISON, R. T.; LEITCH, C. M. Entrepreneurial Learning: Conceptual frameworks and applications. New York: Routledge Taylor \& Francis Group, 2008. p. 1-346 ISBN 0203931920.

HIETANEN, L.; JÄRVI, T. Contextualizing entrepreneurial learning in basic and vocational education. Journal of Enterprising Communities, v. 9, n. 1, p. 45-60, 2015. ISSN 17506204.

HOLCOMB, T. R. et al. Architecture of entrepreneurial learning: exploring the link among heuristics, knowledge, and action. Entrepreneurship Theory and Practice, v. 33, n. 1, p. 167-192, 2009. ISSN 1042-2587.

HUOVINEN, J.; TIHULA, S. Entrepreneurial learning in the context of portfolio entrepreneurship. International Journal of Entrepreneurial Behaviour \& Research, v. 14, n. 3, p. 152-171, 2008. ISSN 1355-2554.

HYTTI, U.; LEMMETYINEN, A. Social entrepreneurship and entrepreneurial learning in the cultural context. Journal of Enterprising Communities, v. 9, n. 1, 2015. ISSN 1750-6204.

INSULANDER, E.; EHRLIN, A.; SANDBERG, A. Entrepreneurial learning in Swedish preschools: possibilities for and constraints on children's active participation. Early Child Development and Care, v. 185, n. 10, p. 1.545-1.555, 2015. ISSN 0300-4430.

JÄMINKI, S. Authentic Entrepreneurial Learning among Students and Entrepreneurs in Entrepreneurial Universities: Insights from the Journeyman Travel Model. In: FINI, R.; GRIMALDI, R. (ed.). Authentic entrepreneurial learning among students and entrepreneurs in entrepreneurial universities: Insights from the journeyman travel model. In: CUNNINGHAM, J.; GUERRERO, M. et al. (ed.). Entrepreneurial Universities: Technology and Knowledge Transfer. Singapura: World Scientific Publishing Co. Pte., 2017. p. 265-296. V. 1. ISBN 9789814733311.

GRIMALDI, R. (ed.). The World Scientific Reference on Entrepreneurship, 2016. p. 265-295. ISBN 978-9814733-39-7. 
JIAO, H.; OGILVIE, D.; CUI, Y. An empirical study of mechanisms to enhance entrepreneurs' capabilities through entrepreneurial learning in an emerging market. Journal of Chinese Entrepreneurship, v. 2, n. 2, p. 196-217, 2010. ISSN 1756-1396.

JONES, O.; MACPHERSON, A.; JAYAWARNA, D. Resourcing the start-up business: Creating dynamic entrepreneurial learning capabilities. New York: Routledge Taylor \& Francis Group, 2014. P. 1-250. ISBN 9780203104569.

JONES, P.; NEWBERY, R.; UNDERWOOD, P. Enhanced entrepreneurial learning through visual experiential learning. In: JONES, P.;MAAS, G. et al. (ed.). Entrepreneurship Education: Contemporary Issues in Entrepreneurship Research. Bingley, Reino Unido: Emerald Group Publishing, 2017. p. 197-211. V. 7. ISBN 20407246.

JUHDI, H. N.; HONG, T. S.; JUHDI, N. Market orientation and entrepreneurial success: Mediating role of entrepreneurial learning intensity. Jurnal Pengurusan, v. 43, p. 27-36, 2015. ISSN 01272713.

KARAJIC, D.; DABIC, M.; CINGULA, M. Interaction among national experts for the Entrepreneurial Learning, within pan-European Network. Procedia - Social and Behavioral Sciences, v. 37, p. 247-256, 2012. ISSN 1877-0428.

KARATAŞ-ÖZKAN, M. Understanding relational qualities of entrepreneurial learning: Towards a multi-layered approach. Entrepreneurship and Regional Development, v. 23, n. 9-10, p. 877-906, 2011. ISSN 08985626.

KIRZNER, I. M. Competition and Entrepreneurship. Chicago: University of Chicago Press, 1973.

KUBBERØD, E.; PETTERSEN, I. B. Exploring situated ambiguity in students' entrepreneurial learning. Education + Training, v. 59, n. 3, p. 265-279, 2017. ISSN 00400912.

KUULUVAINEN, A. Serial entrepreneur and entrepreneurial learning - a case study from Finland. International Journal of Business and Globalisation, v. 4, n. 1, p. 55-70, 2010. ISSN 1753-3627.

KWAN, D. S.; YU, F.-L. T. Entrepreneurial learning and capabilities development of a manufacturing firm in China: The case of the Haier Group. In: YU, F.-L. T.; YAN, H.-D. (ed.). Handbook of East Asian Entrepreneurship. New York: Routledge Taylor \& Francis Group, 2014. p. 391-401. Cap. 32. ISBN 9781317658207.

KWONG, C. C. Y. et al. The role of environment in fostering conductive entrepreneurial learning: Teaching the "art" of entrepreneurship in boot camps. Journal of General Management, v. 38, n. 1, p. 45-71, 2012. ISSN 03063070.

LAGROSEN, S. The Role of Entrepreneurial Learning in Industrial Marketing Success: A Longitudinal Study. EUROPEAN CONFERENCE ON INNOVATION AND ENTREPRENEURSHIP - ECIE, 10., 2015. Genoa, 2015. p. 400-407.

LAGROSEN, S.; JOSEFSSON, P. Social media marketing as an entrepreneurial learning process. International Journal of Technology Marketing, v. 6, n. 4, p. 331-340, 2011a. ISSN 1741-878X.

LAGROSEN, S.; JOSEFSSON, P. Social Media Marketing as an Entrepreneurial Learning Process. ANNUAL EUROMED CONFERENCE OF THE EUROMED ACADEMY OF BUSINESS, 4., 2011. Elounda, 2011b. p. 1.0481.057.

LAGROSEN, Y.; LAGROSEN, S. Entrepreneurial Learning for Quality and Innovation - a Study in the Wellness Industry. EUROPEAN CONFERENCE ON INNOVATION AND ENTREPRENEURSHIP - ECIE, 7., 2012. Santarém: 413-419 p. 2012.

LAGROSEN, S.; JOSEFSSON, P. Entrepreneurial learning for quality and competitiveness: A study in the spa-industry. EUROPEAN CONFERENCE ON INNOVATION AND ENTREPRENEURSHIP - ECIE, 10., 2015. p. 392-399.

LAMONT, L. M. What entrepreneurs learn from experience. Journal of Small Business Management, v. 10, p. 36-41, 1972. ISSN 1540-627X.

LANT, T. K.; MEZIAS, S. J. Managing discontinuous change: a simulation study of organizational learning and entrepreneurship. Strategic Management Journal, v. 11, n. Special Issue, p. 147-179, 1990.

LAVE, J.; WENGER, E. Situated learning: Legitimate peripheral participation. Cambridge: University Press, 1991. ISBN 9780521413084.

LEE, K.; MARRIOTT, S.; LOWE, R. Promoting and Evaluating Entrepreneurial Learning: Assessing the Effectiveness of an Enquiry-Based Approach. EUROPEAN CONFERENCE ON ENTREPRENEURSHIP AND INNOVATION, 4., 2009. Antwerp, 2009. p. 666-673.

LEITE, E. D.; DIAS, C. N. Aprendizagem empreendedora para o desenvolvimento de regiões brasileiras: o fomento de competências na formação acadêmica do Instituto Federal de Brasília. Negócios em Projeção, v. 6, n. 1, p. 204-220, 2015. ISSN 2178-6259.

LEIVA, J. C.; ALEGRE, J.; MONGE, R. The Influence of Entrepreneurial Learning in New Firms' Performance: A Study in Costa Rica. Revista Innovar, v. 24, n. Special Edition, p. 129-140, 2014. ISSN 01215051. 
LIANG, C.-L. K.; DUNN, P. Are entrepreneurs optimistic, realistic, both or fuzzy? Relationship between entrepreneurial traits and entrepreneurial learning. Academy of Entrepreneurship Journal, v. 14, n. 1-2, p. 51-73, 2008. ISSN 1087-9595.

LÖBLER, H. Learning entrepreneurship from a constructivist perspective. Technology Analysis \& Strategic Management, v. 18, n. 1, p. 19-38, 2006. ISSN 0953-7325.

MAN, T. W. Y. Exploring the behavioural patterns of entrepreneurial learning: A competency approach. Education + Training, v. 48, n. 5, p. 309-321, 2006. ISSN 0040-0912.

MAN, T. W. Y. Developing a behaviour-centred model of entrepreneurial learning. Journal of Small Business and Enterprise Development, v. 19, n. 3, p. 549-566, 2012. ISSN 1462-6004.

MARQUES, A. P.; MOREIRA, R.; RAMOS, S. Higher Education, Stakeholders and Collaborative Work for Entrepreneurial Learning. EUROPEAN CONFERENCE ON INNOVATION AND ENTREPRENEURSHIP - ECIE, 9., 2014. p. 320-328.

MCCLELLAND, D. C. A sociedade competitiva realização e progresso social. Rio de Janeiro: Expressão e Cultura, 1972.

MCHENRY, J. The role and management of learning from experience in an entrepreneurial context. In: HARRISON, R. T.; LEITCH, C. M. (ed.). Entrepreneurial Learning: Conceptual Frameworks and Applications. New York: Routledge Taylor \& Francis Group, 2008. p. 72-91. Cap. 4. ISBN 0203931920.

MCKEOWN, I. No More Heroes: Entrepreneurial Learning in the SME management team. Industry and Higher Education, v. 24, n. 6, p. 429-441, 2010. ISSN 0950-4222.

MCKEOWN, I. Entrepreneurial learning in small firm management teams. In: RAE, D.; WANG, C. L. (ed.). Entrepreneurial Learning: New perspectives in research, education and practice. New York: Routledge Taylor \& Francis Group, 2015. p. 178-193. Cap. 9. ISBN 9781317934844.

MIDDLETON, K. W.; DONNELLON, A. Personalizing Entrepreneurial Learning: A Pedagogy for Facilitating the Know-Why. Entrepreneurship Research Journal, v. 4, n. 2, p. 167-204, 2014. ISSN 2194-6175.

MINELLO, I. F.; SCHERER, I. B.; RAMOS, J. L. G. A perspectiva da aprendizagem empreendedora diante do insucesso empresarial. SEMEAD, 17., 2014. São Paulo, 2014.

MINELLO, I. F.; SCHERER, I. B.; RAMOS, J. L. G. A perspectiva da aprendizagem empreendedora diante do insucesso empresarial. ENANPAD, 39., 2015. Belo Horizonte, 2015.

MINNITI, M.; BYGRAVE, W. A Dynamic Model of Entrepreneurial Learning. Entrepreneurship Theory and Practice, v. 25, n. 3, p. 5-16, 2001. ISSN 1042-2587.

MORAES, L. V. D. S.; HOELTGEBAUM, M. Um modelo para a análise do processo de aprendizagem de empreendedores. THIRD INTERNATIONAL CONFERENCE OF THE IBEROAMERICAN ACADEMY OF MANAGEMENT, 3., 2003. São Paulo, 2003. p. 1-20.

MOUSTAGHFIR, K. Entrepreneurial Learning: an Organizational Capability for Effective Higher Education Services. INTERNATIONAL FORUM ON KNOWLEDGE ASSET DYNAMICS - IFKAD, 6., 2011. Tampere, 2011. p. 161-184.

MUHE, A.; TAWE, A. The effect of the entrepreneurial learning design on students' entrepreneurial competence in vocational high schools in Makassar. International Journal of Environmental and Science Education, v. 11, n. 9, p. 3.147-3.159, 2016. ISSN 13063065.

MURPHY, J. Self-Evaluation \& Effectiveness Review for Entrepreneurial Learning and Decision Making. Journal of Enterprising Culture, v. 1, n. 1, p. 109-131, 1993. ISSN 0218-4958.

NICOLINI, D. Practice theory, work, and organization: An introduction. 1. ed. Oxford: University Press, 2012.

NICOLINI, D. Is small the only beautiful? Making sense of 'large phenomena' from a practice-based perspective. In: HUI, A.; SCHATZKI, T. et al. (ed.). The Nexus of Practices: Connections, constellations and practitioners. London: Routledge, 2016. ISBN 9781138675148.

NICOLINI, D.; MONTEIRO, P. The practice approach: for a praxelogy of organizational and management studies. In: LANGLEY, A.; TSOUKAS, H. (ed.). The SAGE handbook of process organization studies. 1. ed. London: Sage Publications, 2016. ISBN 9781446297018.

OWUSU-MANU, D. et al. Redefining Entrepreneurial Learning Paradigms in Developing Countries: A Case Study of Ghana. Industry and Higher Education, v. 27, n. 2, p. 105-116, 2013. ISSN 0950-4222.

PÁSTOR, L.; TAYLOR, L. A.; VERONESI, P. Entrepreneurial learning, the IPO decision, and the post-IPO drop in firm profitability. Review of Financial Studies, v. 22, n. 8, p. 3.005-3.046, 2008. ISSN 08939454.

PENG, X.-Q.; CAI, L. The Relationship between Entrepreneurial Learning and Entrepreneurial Knowledge. 20th INTERNATIONAL CONFERENCE ON MANAGEMENT SCIENCE AND ENGINEERING, 20., 2013. Harbin, 2013. p. 1.314-1.319. 
PIHIE, Z. A. L.; BAGHERI, A.; SANI, Z. H. A. Learning style of university students: Implications for improving entrepreneurial learning paradigm. International Journal of Interdisciplinary Social Sciences, v. 4, n. 4, p. 129-141, 2009. ISSN 18331882.

PITTAWAY, L.; COPE, J. Simulating entrepreneurial learning: Integrating experiential and collaborative approaches to learning. Management Learning, v. 38, n. 2, p. 211-233, 2007. ISSN 13505076.

PITTAWAY, L. et al. Entrepreneurial Learning through Action: A Case Study of the Six-Squared Program. Action Learning: Research and Practice, v. 6, n. 3, p. 265-288, 2009. ISSN 1476-7333.

PITTAWAY, L. et al. The role of entrepreneurship clubs and societies in entrepreneurial learning. International Small Business Journal, v. 29, n. 1, p. 37-57, 2011. ISSN 02662426.

PITTAWAY, L.; THORPE, R. A framework for entrepreneurial learning: A tribute to Jason Cope. Entrepreneurship and Regional Development, v. 24, n. 9-10, p. 837-859, 2012. ISSN 0898-5626.

PLATZEK, B. P.; PRETORIUS, L. Corporate entrepreneurship education: Individual and organizational entrepreneurial learning. INTERNATIONAL ASSOCIATION FOR MANAGEMENT OF TECHNOLOGY - IAMOT, 24., 2015. Cape Town: University of Pretoria, 2015. p. 336-356.

PLATZEK, B. P.; PRETORIUS, L.; WINZKER, D. H. The Vital Entrepreneurial Learning Organization: A Corporate Mindset for Entrepreneurial Change Management. International Journal of Innovation and Technology Management, v. 11, n. 6, 2014. ISSN 0219-8770.

POLITIS, D. The process of entrepreneurial learning: A conceptual framework. Entrepreneurship Theory and Practice, v. 29, n. 4, p. 399-424, 2005. ISSN 1042-2587.

POLITIS, D. Does prior start-up experience matter for entrepreneurs' learning? Journal of Small Business and Enterprise Development, v. 15, n. 3, p. 472-489, 2008.

QIN, H. Effective entrepreneurial learning in the growth process of SMEs. INTERNATIONAL CONFERENCE ON MANAGEMENT OF INNOVATION AND TECHNOLOGY, 4., 2004. Hangzhou, 2004. p. 575-578.

QIN, H.; BAO, G. M. The researches on entrepreneurial learning in the growth process of SMEs. INTERNATIONAL CONFERENCE ON MANAGEMENT SCIENCE \& ENGINEERING, 2004. Harbin, 2004. p. 812-815.

RAE, D. Understanding entrepreneurial learning: a question of how? International Journal of Entrepreneurial Behaviour \& Research, v. 6, n. 3, p. 145-159, 2000. ISSN 1355-2554.

RAE, D. Entrepreneurial learning: a practical model from the creative industries. Education + Training, v. 46, n. 8/9, p. 492-500, 2004a. ISSN 0040-0912.

RAE, D. Practical theories from entrepreneurs' stories: discursive approaches to entrepreneurial learning. Journal of Small Business and Enterprise Development, v. 11, n. 2, p. 195-202, 2004b. ISSN 1462-6004.

RAE, D. Entrepreneurial learning: a narrative-based conceptual model. Journal of Small Business and Enterprise Development, v. 12, n. 3, p. 323-335, 2005a. ISSN 1462-6004.

RAE, D. Mid-career entrepreneurial learning. Education + Training, v. 47, n. 8/9, p. 562-574, 2005b. ISSN 0040-0912.

RAE, D. Entrepreneurial learning: A conceptual framework for technology-based enterprise. Technology Analysis \& Strategic Management, v. 18, n. 1, p. 39-56, 2006. ISSN 09537325.

$\mathrm{RAE}, \mathrm{D}$. The contribution of momentary perspectives to entrepreneurial learning and creativity. Industry and Higher Education, v. 27, n. 6, p. 407-420, Dec. 2013. ISSN 0950-4222.

RAE, D. Towards a momentary perspective in entrepreneurial learning and creativity. In: KNUDSEN, B. T.; CHRISTENSEN, D. R. et al (ed.). Enterprising Initiatives in the Experience Economy: Transforming Social Worlds. New York: Routledge Taylor \& Francis Group, 2014. p. 151-167. Cap. 9. ISBN 978-0415731331.

RAE, D. Entrepreneurial learning: peripherality and connectedness. International Journal of Entrepreneurial Behaviour and Research, v. 23, n. 3, p. 486-503, 2017. ISSN 13552554.

RAE, D.; CARSWELL, M. Understanding entrepreneurial learning: development of a conceptual model and its application in the design of more effective learning experiences. ISBA NATIONAL SMALL FIRMS POLICY AND RESEARCH CONFERENCE, 22., 1999. Leeds, 1999.

RAE, D.; CARSWELL, M. Using a life-story approach in researching entrepreneurial learning: the development of a conceptual model and its implications in the design of learning experiences. Education + Training, v. 42, n. 4/5, p. 220-227, 2000. ISSN 0040-0912.

RAE, D.; CARSWELL, M. Towards a conceptual understanding of entrepreneurial learning. Journal of Small Business and Enterprise Development, v. 8, n. 2, p. 150-158, 2001. ISSN 1462-6004.

RAE, D.; GEE, S.; MOON, R. Creating an Enterprise Culture in a University: The role of an entrepreneurial learning team. Industry and Higher Education, v. 23, n. 3, p. 183-197, 2009. ISSN 0950-4222. 
RAE, D.; GEE, S.; MOON, R. The role of an entrepreneurial learning team in creating an enterprise culture in a university. In: FAYOLLE, A. (ed.). Handbook of Research in Entrepreneurship Education: International Perspectives. Cheltenham - UK: Edward Elgar Publishing, 2010. p. 274-296. Cap. 12. V. 3. ISBN 9781848440968.

RAE, D.; WANG, C. L. Entrepreneurial Learning: New perspectives in research, education and practice. New York: Routledge Taylor \& Francis Group, 2015. p. 1-290. ISBN 9781317934844.

RAMOS, J. L. G. Aprendizagem empreendedora diante do insucesso empresarial: uma perspectiva de empreendedores brasileiros e uruguaios que vivenciaram o fracasso empresarial. 2015. 202p. Dissertação (Mestrado) - Universidade Federal de Santa Maria, Programa de Pós-Graduação em Administração, Santa Maria, 2015.

RAMSGAARD, M. B.; CHRISTENSEN, M. E. Interplay of entrepreneurial learning forms: a case study of experiential learning settings. Innovations in Education and Teaching International, p. 1-10, 2016. ISSN 14703297

ROBERTS, D. C. Rumination to Reflection: The Quest to Re-Visit Entrepreneurial Learning. EUROPEAN CONFERENCE ON RESEARCH METHODOLOGY FOR BUSINESS AND MANAGEMENT STUDIES, 10., 2011. Caen, 2011. p. 614-620.

ROMANO, A.; PASSIANTE, G.; DEL VECCHIO, P. Knowledge-intensive Entrepreneurship in Innovation Ecosystems: towards the conceptual model of a Regional Entrepreneurial Learning Center. INTERNATIONAL FORUM ON KNOWLEDGE ASSET DYNAMICS, 9., 2014. Matera, 2014. p. 3.033-3.050.

ROSSIGNOLI, F.; LIONZO, A.; LASSINI, U. Entrepreneurial learning in family SMEs: The role of family in communities of practice. EUROPEAN CONFERENCE ON KNOWLEDGE MANAGEMENT - ECKM, 17., 2016. Belfast, 2016, p. 771-779.

RUSK, M.; MCGOWAN, P. Entrepreneurial Learning in Context: An Exploration of Learning Models in Different Domains. EUROPEAN CONFERENCE ON INNOVATION AND ENTREPRENEURSHIP - ECIE, 10., 2015. Genoa, 2015. p. 600-608.

SACRAMENTO, P. M. Inovação e aprendizagem empreendedora: estudo de casos múltiplos em empresas turísticas de pequeno e médio porte da cidade de Aracaju. 2013. 192p. Dissertação (Mestrado) - Universidade Federal do Sergipe, Programa de Pós-Graduação em Administração, São Cristóvão, 2013.

SALUTO, P.; GUELFI, S.; NORESE, M. F. An integration of theoretical knowledge, day by day experience and multicriteria methods to support the entrepreneurial learning process. Measuring Business Excellence, v. 20, n. 1, p. 12-20, 2016. ISSN 1368-3047.

SANZ-VELASCO, S. Entrepreneurial learning: Developing opportunities and business models. 2006. 60p. Thesis (Doctoral) - Institutionen för Teknikens Ekonomi och Organisation, Chalmers University of Technology, Göteborg, 2006.

SAY, J. B. A treatise on political economy. 6 th ed. New York: Augustus M. Kelley, 1971.

SCARMOZZINO, E.; CORVELLO, V.; GRIMALDI, M. Entrepreneurial learning through online social networking in high-tech startups. International Journal of Entrepreneurial Behaviour and Research, v. 23, n. 3, p. 406-425, 2017. ISSN 13552554.

SCHERER, R. F.; ADAMS, J. S.; WIEBE, F. A. Social learning theory as a conceptual framework for entrepreneurship research: The role of observational learning. In: ROBERTS, G. B.;LASHER, H. et al. International Council for Small Business, Boston, p. 243-249, 1988.

SCHOUT, H.; HARKEMA, S. Entrepreneurial Learning: Practice as a Source for Learning and Business Success. EUROPEAN CONFERENCE ON INNOVATION AND ENTREPRENEURSHIP, 5., 2010. Athens, 2010. p. 771-774.

SCHUMPETER, J. A. Teoria do desenvolvimento econômico: uma investigação sobre lucros, capital, crédito, juro e o ciclo econômico. São Paulo: Nova Cultural, 1997. p. 1-240 ISBN 85-351-0915-3.

SECUNDO, G. et al. Students' creativity and entrepreneurial learning for developing corporate entrepreneurship. In: SCHIUMA, G.; LERRO, A. (ed.). Integrating Art and Creativity into Business Practice, 2017. p. 89-116. ISBN 9781522520511.

SECUNDO, G. et al. Enhancing corporate entrepreneurship through an entrepreneurial learning approach: turning students' ideas into entrepreneurial practice. 10th INTERNATIONAL FORUM ON KNOWLEDGE ASSET DYNAMICS - IFKAD, 10., 2015. Bari, 2015a.

SECUNDO, G. et al. Entrepreneurial learning dynamics for technology driven entrepreneurship: An integrative framework. EUROPEAN CONFERENCE ON KNOWLEDGE MANAGEMENT - ECKM, 16., 2015. Udine, 2015b. p. 676-683. 
SECUNDO, G. et al. Activating entrepreneurial learning processes for transforming university students' idea into entrepreneurial practices. International Journal of Entrepreneurial Behaviour and Research, v. 23, n. 3, p. 465-485, 2017. ISSN 13552554.

SECUNDO, G.; SCHIUMA, G.; PASSIANTE, G. Entrepreneurial learning dynamics in knowledge-intensive enterprises. International Journal of Entrepreneurial Behaviour and Research, v. 23, n. 3, p. 366-380, 2017. ISSN 13552554.

SEUNEKE, P.; LANS, T.; WISKERKE, J. S. C. Moving beyond entrepreneurial skills: Key factors driving entrepreneurial learning in multifunctional agriculture. Journal of Rural Studies, v. 32, n. 2013, p. 208-219, 2013. ISSN 07430167.

SHANE, S.; VENKATARAMAN, S. The Promise of Entrepreneurship as a Field of Research. The Academy of Management Review, v. 25, n. 1, p. 217-226, 2000. ISSN 03637425.

SILVA, J. C. P. D. Aprendizagem e competências empreendedoras: estudo com gestores de tecnologia da informação na região metropolitana de Fortaleza-CE. 2014. 126p. Dissertação (Mestrado Profissional em Administração e Controladoria) - Universidade Federal do Ceará, Faculdade de Economia, Administração, Atuária, Contabilidade e Secretariado Executivo, Fortaleza, 2014.

SJOEVOLL, J.; PEDERSEN, O. Links between Educational Programmes, Local Recourses and Entrepreneurial Learning. INTERNATIONAL CONFERENCE OF EDUCATION, RESEARCH AND INNOVATION, 3., 2010. Madrid, 2010.

SMILOR, R. W. Entrepreneurship: Reflections on a subversive activity. Journal of Business Venturing, v. 12, n. 5, p. 341-346, 1997. ISSN 0883-9026.

SOETANTO, D. Networks and entrepreneurial learning: coping with difficulties. International Journal of Entrepreneurial Behaviour and Research, v. 23, n. 3, p. 547-565, 2017. ISSN 13552554.

SPITERI, S.; MARINGE, F. EU entrepreneurial learning: perspectives of university students. Journal of Enterprising Communities, v. 8, n. 1, p. 51-70, 2014. ISSN 1750-6204.

SULLIVAN, R. Entrepreneurial learning and mentoring. International Journal of Entrepreneurial Behaviour \& Research, v. 6, n. 3, p. 160-175, 2000. ISSN 1355-2554.

TÄKS, M.; TYNJÄLÄ, P.; KUKEMELK, H. Engineering students' conceptions of entrepreneurial learning as part of their education. European Journal of Engineering Education, v. 41, n. 1, p. 53-69, 2016. ISSN 03043797.

TAYLOR, D. W.; THORPE, R. Entrepreneurial learning: a process of co-participation. Journal of Small Business and Enterprise Development, v. 11, n. 2, p. 203-211, 2004. ISSN 1462-6004.

TERZIEVA, L. Entrepreneurial learning and communities of practice: The case of the cross-border cultural tourism development Bulgaria- Romania. In: ATELJEVIC, J.; TRIVIĆ, J. (ed.). Economic Development and Entrepreneurship in Transition Economies: Issues, Obstacles and Perspectives. Switzerland: Springer International Publishing, 2016. p. 271-286. Cap. 15. ISBN 9783319288567.

THEODORAKOPOULOS, N. et al. Diversifying into technical clothing manufacture as entrepreneurial learning: A situated learning theory perspective. Journal of Manufacturing Technology Management, v. 25, n. 5, p. 676-693, 2014. ISSN 1741-038X.

TOUTAIN, O. et al. Role and impact of the environment on entrepreneurial learning. Entrepreneurship \& Regional Development, v. 29, n. 9-10, p. 869-888, 2017.

TRAGAZIKIS, P.; KIRGINAS, S.; GOUSCOS, D. Digital games for entrepreneurial learning, innovation and creativity: examples and evaluation criteria. International Journal of Innovation and Regional Development, v. 4, n. 3-4, p. 314-337, 2012. ISSN 1753-0660.

TRANFIELD, D.; DENYER, D.; SMART, P. Towards a Methodology for Developing Evidence-Informed Management Knowledge by Means of Systematic Review. British Journal of Management, 14, p. 207-222, 2003. ISSN 1467-8551.

TUCKER JR., L. R. Entrepreneurial Learning Experience: The Academic Responsibility. Journal of Business Education, v. 56, n. 4, p. 132-135, 1981.

VAN VUUREN, W. et al. Entrepreneurial learning and the IBM universities business challenge: an experiential learning perspective. In: FAYOLLE, A.; REDFORD, D. T. (ed.). Handbook on the Entrepreneurial University. Cheltenham, UK: Edward Elgar Publishing Limited, 2014. p. 392-413. Cap. 17. ISBN 9781781007013.

WALSH, G. S.; CUNNINGHAM, J. A. Regenerative failure and attribution: Examining the underlying processes affecting entrepreneurial learning. International Journal of Entrepreneurial Behaviour and Research, v. 23, n. 4, p. 688-707, 2017. ISSN 13552554.

WANG, C. L.; CHUGH, H. Entrepreneurial Learning: Past Research and Future Challenges. International Journal of Management Reviews, v. 16, n. 1, p. 24-61, 2014. ISSN 14608545. 
WANG, C. L.; CHUGH, H. Entrepreneurial learning: Past research and future challenges. In: RAE, D.; WANG, C. L. (Ed.). Entrepreneurial Learning: New perspectives in research, education and practice. New York: Routledge Taylor \& Francis Group, 2015. p. 11-44. Cap. 2. ISBN 9781317934844.

WEE, K. N. L. A problem-based learning approach in entrepreneurship education: promoting authentic entrepreneurial learning. International Journal of Technology Management, v. 28, n. 7/8, p. 685-701, 2004. ISSN 02675730.

WEI, J.; GONG, L.; LIU, C. Entrepreneurial Learning Based on Critical Incidents: Influences of Learning Styles. INTERNATIONAL CONFERENCE ON STRATEGIC MANAGEMENT, 2009. Chengdu, 2009. p. 427-433.

WENGER, E. Communities of practice: Learning, meaning, and identity. Cambridge: University Press, 1998. ISBN 978-0521663632.

WILKINSON, M. A Sociocultural-Historical Perspective on Entrepreneurial Learning: The Case of a Novice Entrepreneur. INTERNATIONAL SEMINAR QUALITY MANAGEMENT IN HIGHER EDUCATION, 6., 2010. Tulcea, 2010. p. 715-718.

XIAO, Q.; ZHUANG, W.-L.; CAO, Z.-P. A situated model of entrepreneurial learning and entrepreneurs' innovation propensity and its application in China's transitional economy. Entrepreneurial Strategy Innovation and Sustainable Development. Chengdu, 2007. p. 174-181.

$\mathrm{XU}$, J.; LU, Y. Impacts of entrepreneurial network on entrepreneurial learning. INTERNATIONAL CONFERENCE ON INFORMATION MANAGEMENT, INNOVATION MANAGEMENT AND INDUSTRIAL ENGINEERING - ICIII, 3., 2010. Kunming, 2010. p. 401-404.

YANG, J.-P.; CHEN, T.-T.; TANG, L.-B. The effect of entrepreneurial network on new venture growth: The regulatory role of entrepreneurial learning. INTERNATIONAL ASIA CONFERENCE ON INDUSTRIAL ENGINEERING AND MANAGEMENT INNOVATION - IEMI, 2013. Beijing, 2013. p. 1.699-1.708.

YOUNG, J. E.; SEXTON, D. L. Entrepreneurial Learning: A Conceptual Framework. Journal of Enterprising Culture, v. 5, n. 3, p. 223-248, 1997. ISSN 0218-4958.

ZAMPIER, M. A. Desenvolvimento de competências empreendedoras e processos de aprendizagem empreendedora: estudo de casos de MPE's do setor educacional. 2010. 298p. Dissertação (Mestrado) - Universidade Federal do Paraná, Centro de Pesquisa e Pós-Graduação em Administração, Curitiba, 2010.

ZAMPIER, M. A.; TAKAHASHI, A. R. W. Competências empreendedoras e processos de aprendizagem empreendedora: modelo conceitual de pesquisa Cadernos EBAPE.BR, v. 9, n. Edição Especial, p. 564-585, jul. 2011. ISSN 1679-3951.

ZAMPIER, M. A.; TAKAHASHI, A. R. W. Aprendizagem e competências empreendedoras: estudo de casos de MPE's do setor educacional. ENCONTRO DE ESTUDOS SOBRE EMPREENDEDORISMO E GESTÃO DE PEQUENAS EMPRESAS - EGEPE, 7., 2012. Florianópolis, 2012.

ZAMPIER, M. A.; TAKAHASHI, A. R. W. Competências e aprendizagem empreendedora em MPE's educacionais. Revista Pensamento Contemporâneo em Administração, v. 8, n. 3, p. 1-22, 2014. ISSN 1982-2596. ZHANG, X. The Impact of Entrepreneurial Learning on Entrepreneurial Performance: The Mediating Role of Entrepreneurial Competencies. In: XIE, A.; HUANG, X. (ed.). Advances in Electrical Engineering and Automation. New York: Springer, 2012. p. 229-234. ISBN 978-3-642-27950-8.

ZHENG, K. The Entrepreneurial Learning Process And Influential Factors of Entrepreneurs in Business Model Construction. INTERNATIONAL CONFERENCE ON EDUCATION, MANAGEMENT AND COMPUTING TECHNOLOGY - ICEMCT, 30., 2015. Tianjin, 2015. p. 1.382-1.386 p.

ZHIYUAN, L.; SHUKUAN, Z. Networking ties and entrepreneurial learning: An empirical investigation of Chinese enterprisers. INTERNATIONAL CONFERENCE ON NETWORKING AND DIGITAL SOCIETY - ICNDS, 2., 2010. Wenzhou, 2010. p. 104-108. 